\title{
1 Public Health: Konzepte, Disziplinen und Handlungsfelder
}

\author{
Matthias Egger, Oliver Razum, Anita Rieder
}

In diesem einführenden Kapitel lernen wir die zentralen Begriffe, Konzepte, Disziplinen und Handlungsfelder von Public Health kennen. Ein Blick in das 19.Jh. zeigt, dass Public Health zu Beginn überraschenderweise weniger mit der Medizin als mit dem Ingenieurwesen zu tun hatte. Die Geschichte macht auch verständlich, warum heute der englische Begriff „Public Health“ auch im Deutschen gebräuchlich ist. Public Health und Medizin unterscheiden sich in ihrer Sicht auf Krankheit und Gesundheit. Anders als im medizinischen Denken steht in Public Health die Entstehung von Gesundheit (Salutogenese) und nicht die Entstehung von Krankheit (Pathogenese) im Mittelpunkt. Zu den Kernthemen von Public Health gehört u. a. die gesundheitliche Ungleichheit zwischen verschiedenen Bevölkerungsgruppen, z. B. die Ungleichheit im Zusammenhang mit der sozialen Schichtzugehörigkeit und dem Geschlecht. Bei vielen Public-Health-Fragen spielen auch ethische Aspekte eine Rolle. Während in der Medizinethik die Arzt-Patient-Beziehung im Mittelpunkt steht, ist es in der Public-Health-Ethik das Verhältnis zwischen den Institutionen und den Bürgerlnnen. Wir schließen das Kapitel mit einem kritischen Blick auf die Public Health Genomics und ihrem Versprechen einer individualisierten Prävention.

Schweizerische Lernziele: $\mathrm{CPH}$ 1-3, CPH 28-34

\subsection{Definition}

Unter Public Health verstehen wir eine von der Gesellschaft organisierte, gemeinsame Anstrengung, mit dem Ziel der

- Erhaltung und Förderung der Gesundheit der gesamten Bevölkerung oder von Teilen der Bevölkerung,

- Vermeidung von Krankheit und Invalidität,

- Versorgung der Bevölkerung mit präventiven, kurativen und rehabilitativen Diensten.

Im deutschsprachigen Raum wird synonym auch etwas umständlich von der öffentlichen Gesundheitspflege gesprochen. Der Begriff der Volksgesundheit ist durch den Nationalsozialismus belastet (s. Kap. 1.2) und wird deshalb nicht verwendet. Aus den genannten Gründen ist der englische Begriff „Public Health“ auch im Deutschen gebräuchlich. Im Gegensatz zur kurativen Individualmedizin richtet Public Health den Blick auf die gesamte Bevölkerung oder auf Bevölkerungsgruppen und beschäftigt sich hier mit ethisch (s. Kap. 1.6) und ökonomisch (s. Kap. 2.5.1) vertretbaren Maßnahmen der Gesundheitsförderung, der Krankheitsprävention und der Versorgung. 
Handlungsfelder von Public Health sind

- die wissenschaftliche Forschung an universitären Instituten: In der Schweiz geschieht das z.B. an Instituten für Sozial- und Präventivmedizin und dem Schweizerischen Tropen- und Public Health-Institut (Swiss TPH). In Österreich findet Public-Health-Forschung vor allem an den medizinischen Universitäten statt. Zentren für Public Health kooperieren dabei oftmals mit Institutionen des Öffentlichen Gesundheitswesens oder umgekehrt. In Deutschland forschen Public-Health-Wissenschaftler v. a. an gesundheitswissenschaftlichen Instituten sowie an medizinischen Instituten mit Public-Health-Ausrichtung.

- die Praxis in den Public-Health-Institutionen: In der Schweiz sind hierfür z. B. die kantonalen Gesundheitsämter und das Bundesamt für Gesundheit (BAG) zuständig. In Österreich ist das Bundesministerium für Gesundheit und Frauen (BMGF) für die gesundheitliche Rahmengesetzgebung verantwortlich. Die Gesundheit Österreich $\mathrm{GmbH}$ ist mit der Strukturplanung, Gesundheitsförderung und Qualitätssicherung im Gesundheitswesen beauftragt. In Deutschland ist das Robert Koch-Institut die zentrale Einrichtung auf dem Gebiet der Krankheitsüberwachung und -prävention.

- die Gesundheits- und Sozialpolitik, die durch Verordnungen und Gesetze das Gesundheitswesen steuert und gesundheitsfördernde Arbeits- und Lebensbedingungen schafft.

Zu den Aufgaben von Public-Health-Institutionen gehört es, die Gesundheit der Bevölkerung zu schützen und zu überwachen (Surveillance), etwa im Zusammenhang mit Infektionskrankheiten (s. Kap. 9.2.2), der Lebensmittelsicherheit (s. Kap. 6.2.4), der Sicherheit am Arbeitsplatz (s. Kap. 7.2.1) oder der Luftverschmutzung (s. Kap. 6.4.3). Darüber hinaus sind sie u.a. für die Erarbeitung und Durchführung von Impfprogrammen (s. Kap. 9.4.1), Screening-Programmen (s. Kap. 4.5.4) und Aufklärungskampagnen (s. Kap. 4.1.2) zuständig. Hierbei arbeiten Fachleute verschiedenster Disziplinen aktiv zusammen (s. Kap. 1.4). Beispiele für gesundheitspolitische Maßnahmen sind Rauchverbote in öffentlichen Räumen (s. a. Kap. 4.2.2) und die laufenden Bestrebungen, Gesundheitsförderung und Prävention zu stärken, so in Deutschland z. B. durch das 2015 in Kraft getretene Präventionsgesetz (PrävG, s. Kap. 4.1.3). In Österreich wurde die Gesundheitsförderung durch das 1998 verabschiedete Gesundheitsförderungsgesetz maßgeblich beeinflusst.

Der Master of Public Health (MPH) ist ein international anerkannter akademischer Grad, der im angelsächsischen Raum (z. B. an der geschichtsträchtigen London School of Hygiene \& Tropical Medicine oder an den Schools of Public Health nordamerikanischer Universitäten), aber auch an verschiedenen Hochschulen in Deutschland, Österreich und der Schweiz erworben werden kann. Ein MPH-Studium ist in der Schweiz Teil der Weiterbildung zum Facharzt in Prävention und Gesundheitswesen. In Deutschland kann der Facharzt für Öffentliches Gesundheitswesen und der Facharzt für Hygiene und Umweltmedizin erworben werden. In Österreich 
wurde der Facharzt für Sozialmedizin im Jahr 2016 durch eine Facharztausbildung Public Health abgelöst. In allen drei Ländern gibt es darüber hinaus einen Facharzttitel im Bereich der Arbeitsmedizin, in Deutschland noch die Zusatzbezeichnung Betriebsmedizin.

\subsection{Geschichtliche Notizen \\ Matthias Egger, Lukas Fenner}

\section{Die soziale Frage}

Die Entwicklung der modernen Public Health ist eng mit der sozialen Reformbewegung im 19. Jahrhundert verbunden, die darauf abzielte, die soziale Lage der Arbeiter und ihrer Familien zu verbessern. Abb. 1.1 illustriert die Lebensumstände der damaligen Arbeiterschaft am Beispiel einer Behausung in London. Angesichts dieser

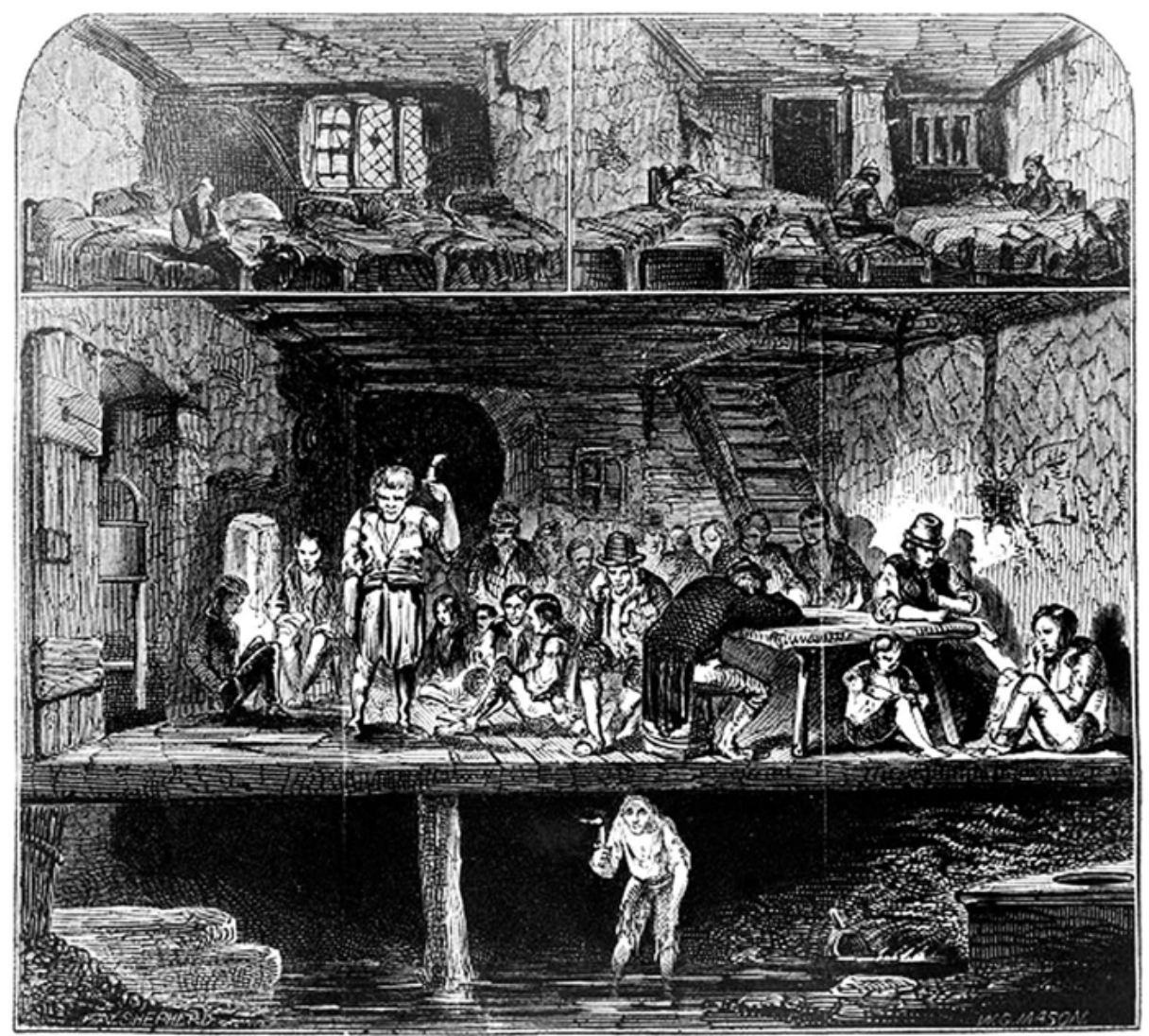

Abb. 1.1: Eine Londoner Behausung an der Field Lane im 19. Jahrhundert. Die Notdurft wurde am Kanal verrichtet (Quelle: Wellcome Images. https://wellcomeimages.org/). 
Zustände überrascht es nicht, dass London und andere europäische Städte zu jener Zeit immer wieder von Choleraepidemien heimgesucht wurden (s.a. Abb. 1.2) und dass dort auch die Tuberkulose grassierte. Im Zentrum der angestrebten Reformen standen die Verbesserung der sanitären Bedingungen in den Städten und der Verhältnisse am Arbeitsplatz. In England förderte der Public Health Act von 1848 den Bau von Wasserleitungen und Kanalisationsanlagen (s.a. Kap. 6.2.1). In Berlin trieb der Pathologe und Sozialreformer Rudolf Virchow (1821-1902, s. a. Kap. 2.1.1) den Bau von zentraler Wasserversorgung und Kanalisation voran, während in München Max von Pettenkofer (1818-1901) hierbei die treibende Kraft war.

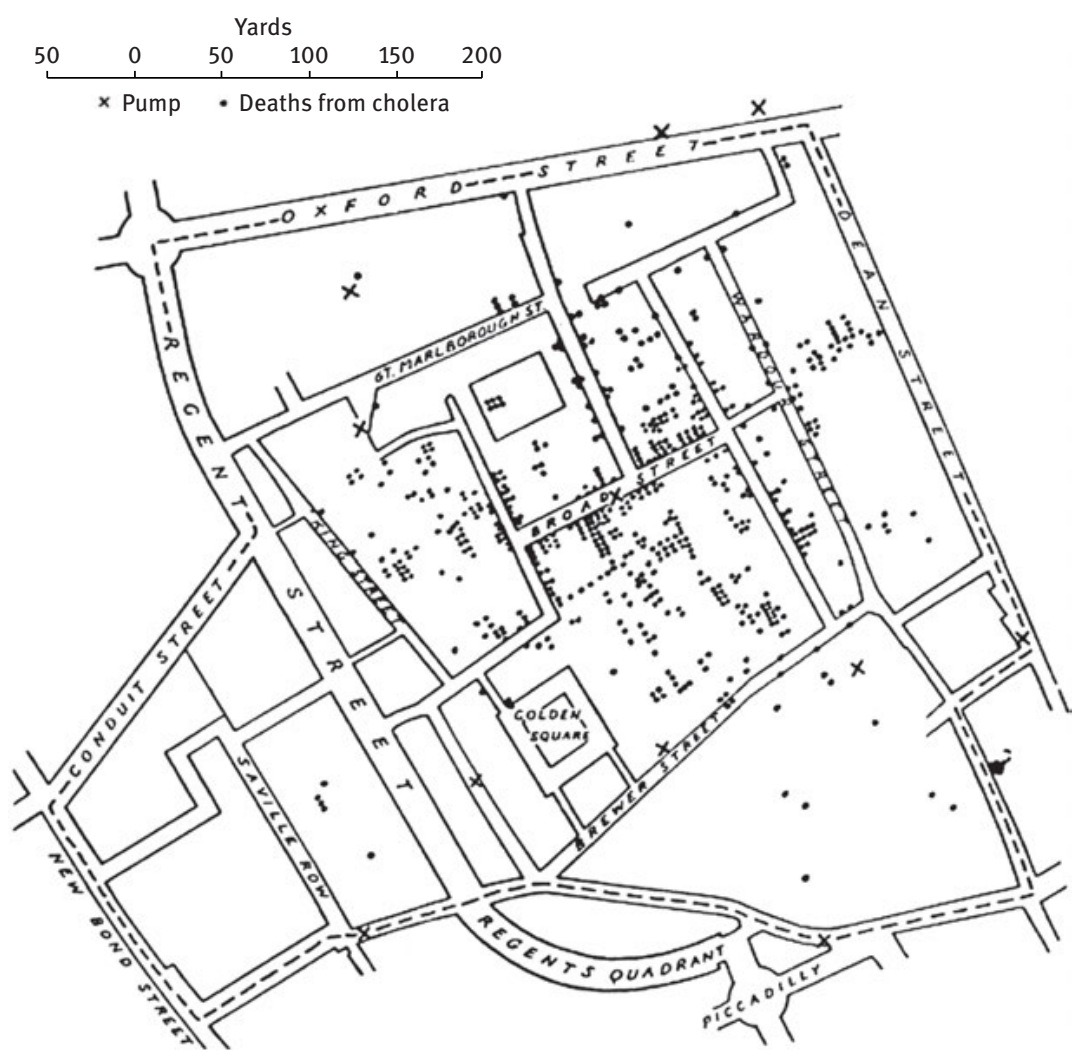

Abb. 1.2: Karte der Cholera-Todesfälle im Rahmen der Epidemie von 1854, die rund um die BroadStreet-Wasserpumpe auftraten. Der Epidemiologe John Snow (1813-1858, s. a. Kap. 2.1.1) folgerte hieraus, dass die Cholera durch verschmutztes Trinkwasser übertragen wird und entfernte den Pumpengriff, um weitere Ansteckungen zu verhindern. (Rekonstruktion der Karte nach Snows Angaben durch den medizinischen Geografen E.W. Gilbert, 1958) (Quelle: Gilbert E.W. Pioneer map and health and disease in England. Geographical Journal 1958; 124(2): 172-183). 


\section{Hygiene und Sozialhygiene}

Pettenkofer hatte ab 1865 den ersten Lehrstuhl für Hygiene in Deutschland inne. Zentrale Themen dieses neuen medizinischen Fachgebietes waren die Verhütung von Krankheiten und die Förderung der Gesundheit der Bevölkerung. Pettenkofers besonderes Interesse galt dabei der physikalischen und chemischen Umwelt. Er gilt deshalb als Wegbereiter der Umweltepidemiologie und Umweltmedizin (s. Kap. 5). Mit der Entdeckung der Bakterien und dem im Jahr 1882 durch Robert Koch (1843-1910) erfolgten Nachweis von Mycobacterium tuberculosis als einzigen, eindeutig identifizierbaren Krankheitserreger der Tuberkulose wurde die Bakteriologie zur führenden Gesundheitswissenschaft des ausgehenden 19. Jahrhunderts. Damit war die Debatte um die Frage, wodurch Krankheiten verursacht werden, jedoch noch nicht abgeschlossen. Die von Alfred Grotjahn (1869-1931) begründete Sozialhygiene stellte die monokausale Erklärung der Entstehung von Infektionskrankheiten in Frage und betonte die Wichtigkeit von gesellschaftlichen Einflüssen, wie z. B. von engen und unhygienischen Wohnverhältnissen, schlechter Ernährung oder niedrigem Einkommen auf die Krankheitsentstehung (s. Abb. 1.3, mit einem Beispiel einer Studie aus dieser Zeit). Grotjahn vertrat allerdings als Mitglied der Gesellschaft für Rassenhygiene auch eugenische Vorstellungen (s.u.). Zu Beginn des 20. Jahrhunderts war Deutschland auf dem Gebiet der Hygiene führend, was sich u. a. daran zeigte, dass die erste Internationale Hygiene-Ausstellung 1911 in Dresden von mehr als fünf Mio. Menschen (!) besucht wurde. Die Schaffung von kommunalen Gesundheitsämtern in Deutschland (heute oft als Fachdienst Gesundheit bezeichnet) ist ein bleibender Verdienst jener Zeit.

Aber auch in der Schweizer Hauptstadt Bern gelang es beispielsweise, die Zahl der Tuberkulose-Toten zu senken, schon bevor ab Mitte des 20. Jahrhunderts eine wirksame

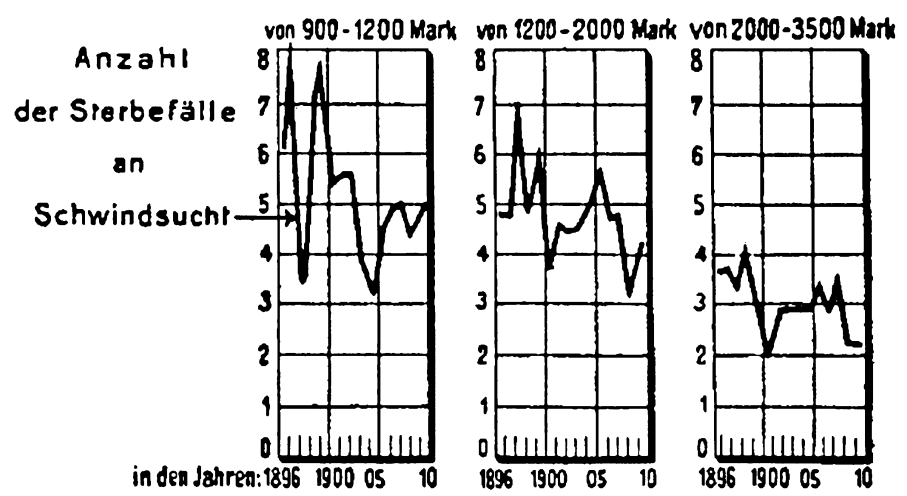

Abb. 1.3: Beispiel einer sozialhygienischen Studie, die zu Beginn des 20. Jh. in Deutschland durchgeführt wurde. Die Grafik zeigt das Einkommen (in Mark) und die Tuberkulosesterblichkeit (pro 1.000 Einwohner) in Hamburg in den Jahren zwischen 1896 und 1910. Menschen mit höherem Einkommen wiesen eine deutlich niedrigere Sterblichkeit an Tuberkulose auf als Menschen aus niedrigeren Einkommensschichten.

(Quelle: Mosse M, Tugendreich G. Krankheit und Soziale Lage, 1913). 
medizinische Behandlung zur Verfügung stand. Zum frühen Erfolg in der Tuberkulosebekämpfung führten neben dem medizinischen Fortschritt auch andere Faktoren, wie die allgemeinen Verbesserungen der Lebensumstände und Public-Health-Maßnahmen, die auf Krankheitsprävention und Gesundheitsförderung zielten (s. Box 1.2.1). So wurden in Bern öffentliche Sanatorien für Tuberkulose-Erkrankte und Freiluftschulen für gefährdete Kinder errichtet, etwas später kamen Screening-Untersuchungen (s. Kap. 4.5) mit Hilfe von Röntgen-Apparaten und Tuberkulin-Hauttest hinzu.

Box 1.2.1: Lebensbedingungen und Tuberkulose-Sterblichkeit in der Stadt Bern im 19. und 20. Jahrhundert.

Die früher auch als Phthise, Schwindsucht, Auszehrung, weiße Pest oder bleiches Sterben bezeichnete bakterielle Infektionskrankheit Tuberkulose wird durch Tröpfcheninfektion übertragen. Erreger ist das Mycobacterium tuberculosis. Das Bakterium wurde 1882 durch den deutschen Mediziner Robert Koch entdeckt. Es verursachte Ende des 19. und im frühen 20. Jahrhundert in allen europäischen Ländern bis zu einem Viertel aller Todesfälle. Um 1900 lag die Infektions-Prävalenz bei Jugendlichen in der Schweiz bei $100 \%$. Die Erkrankung wurde als „Volkskrankheit“ betrachtet und gehörte zu den „gewöhnlichen Schattenseiten des Lebens“. In der Stadt Bern (Schweiz) variierte die Tuberkulose-Sterblichkeit stark zwischen den einzelnen Stadtteilen. So war sie in Teilen der Altstadt - insbesondere im Schwarzen Quartier - fast doppelt so hoch wie in der übrigen Innenstadt (550/100.000 gegenüber 327/100.000). Die Belegung der Wohnungen war in diesem ehemalige Handwerker- und Arbeiterstadtteil besonders hoch (2,2 Personen pro Raum; andere Stadtgebiete: 1,4 Personen). Die Wohnungsgröße war deutlich geringer ( $18 \mathrm{~m}^{3}$ pro Person gegenüber $31 \mathrm{~m}^{3}$ ), was die Übertragung der Tuberkulose begünstigte. Auch der durch Verunreinigung von Wasser und Lebensmitteln ausgelöste Typhus war im Slum-ähnlichen Schwarzen Quartier wesentlich verbreiteter. Eine statistische Auswertung der Verhältnisse um 1896 („Wohnungsenquête“) belegt die miserablen Zustände: $77 \%$ der Wohnungen verfügten über keine eigene Toilette, $74 \%$ hatten kein fließendes Wasser, $11 \%$ waren feucht und baulich unbefriedigend.

Nicht nur in der Schweiz, sondern auch in anderen europäischen Ländern ging die Tuberkulose-Sterblichkeit jedoch seit Mitte des 19. Jahrhunderts stetig zurück. So nahm sie z. B. in Bern zwischen 1856 und 1950 um das Zehnfache ab, und zwar lange bevor eine effektive Therapie zur Verfügung stand. Zum Erfolg trugen die allgemeine Verbesserungen der Lebensumstände, eine bauliche Aufwertung der Häuser, insbesondere der Belüftung, sowie andere Public-Health-Maßnahmen bei (s. Abb.). Am Ende des 19. Jahrhunderts wurden in der Schweiz die ersten öffentlichen Volksheilstätten für Lungenkranke eröffnet. Es folgten Freiluftschulen für Tuberkulose gefährdete Kinder im Jahr 1923. Ab 1930 wurde bei allen Schulkindern ein Tuberkulin-Hauttest durchgeführt, ab 1940 zusätzlich noch systematische Röntgen-Thorax-Kontrollen. Für lange Zeit bestand die Behandlung der Tuberkulose vorwiegend aus Bettruhe, Freiluft-Liegekuren, Sonnenkuren (Heliotherapie), guter Ernährung und der Kollapstherapie (Herstellung eines künstlichen Pneumothorax ${ }^{1}$ ). Das erste gegen Tuberkulose wirksame Antibiotikum, Streptomycin, konnte dann ab 1948 klinisch eingesetzt werden.

1 Künstlicher Pneumothorax: Die erkrankte Lungenseite wurde durch das Einbringen von Stickstoff oder gefilterter Luft in den Pleuraspalt stillgelegt, um den Heilungsprozess bei offener Tuberkulose zu unterstützen. 


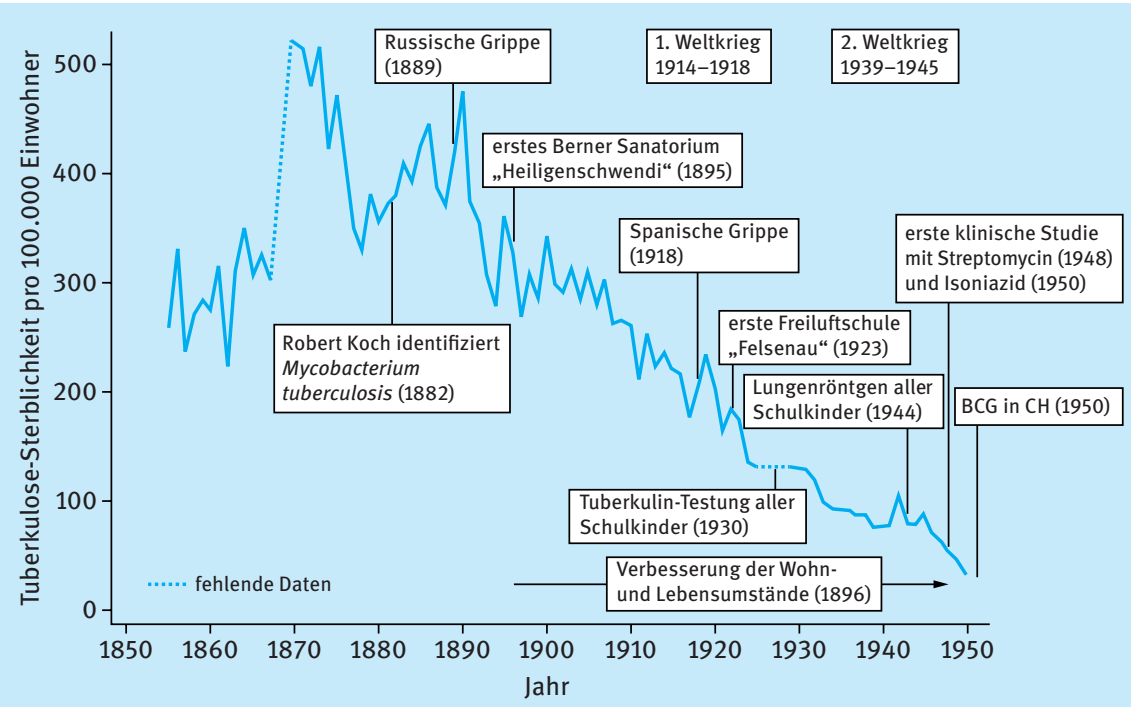

Während die Tuberkulose in Ländern mit hohem Einkommen heute selten geworden ist (Schweiz: <7 Fälle/100.000 Einwohner), grassiert die „Krankheit der Armen“ in anderen Regionen immer noch stark (Afrika: 281/100.000). Nach Schätzung der WHO gab es 2015 weltweit mehr als zehn Mio. neuer Krankheitsfälle, an Tuberkulose starben in diesem Zeitraum 1,5 Mio. Menschen.

BCG: Bacille Calmette-Guérin, abgeschwächt virulentes Bakterium, das die Basis für einen ersten Tuberkulose-Lebendimpfstoff bildete;

WHO: World Health Organization, Weltgesundheitsorganisation

\section{Eugenik und Nationalsozialismus}

Die Sozialhygiene war eng mit der Eugenik oder Rassenhygiene verbunden. Hierunter verstand man die Anwendung von Erkenntnissen aus der Humangenetik auf die Bevölkerung mit dem Ziel, die Fortpflanzung von „Gesunden“ zu fördern und dadurch den Anteil an Menschen mit „positiven“ Erbanlagen zu erhöhen. Auch der Sozialhygieniker Grotjahn (s. o.) war Mitglied der Gesellschaft für Rassenhygiene und befürwortete die Zwangssterilisierung von Menschen mit körperlicher oder geistiger Behinderung, von Menschen mit Epilepsie und von Alkoholkranken. Die Eugenik geht auf den englischen Naturforscher Francis Galton (1822-1911) zurück, einem Vetter Charles Darwins. Die ersten eugenisch motivierten Sterilisationen wurden in Europa bereits um 1890 durch den Psychiater und Ameisenforscher Auguste Forel (1848-1931) in der Psychiatrischen Universitätsklinik Burghölzli in Zürich durchgeführt. Nach ihrer Machtergreifung im Jahr 1933 setzten die Nationalsozialisten das auf den Ideen der Rassenhygiene beruhende, menschenverachtende Ziel eines ,rassenreinen arischen Volkskörpers“ konsequent und mit unglaublicher Grausamkeit mit Hilfe von Massensterilisierungen, Massentötungen und Genozid durch. Weniger bekannt sind andere Aspekte der nationalsozialistischen Gesundheitspolitik, wie Maßnahmen 
gegen das Rauchen, Verbote von petrochemischen Kanzerogenen und der Schutz vor Asbest am Arbeitsplatz (s.a. Kap. 7.7). Tabak galt dabei nicht nur als Krebserreger und Ursache von Herzkrankheiten, sondern auch als Rassengift, das die Fruchtbarkeit und Arbeitskraft der Menschen einschränkt. Nichtrauchen war daher eine Gesundheitspflicht. Das Rauchen in der Öffentlichkeit sowie die Tabakwerbung wurden eingeschränkt oder verboten. Eine Abbildung in Kap. 1 auf unserer Lehrbuch-Homepage zeigt ein Werbeplakat aus einer Kampagne gegen das Rauchen aus dem Jahr 1941. Die unglückliche Verbindung mit dem Nationalsozialismus, die sich im englischen Sprachraum in Begriffen wie „nicoNazi“ oder „health facism“ niederschlägt, belastet die Tabakprävention noch heute. Wie wenig erfolgreich die nationalsozialistische Tabakpolitik jedoch war, zeigt sich u. a. dadurch, dass die „Amis“ (d. h. die amerikanischen Zigaretten) Deutschland nach dem Zweiten Weltkrieg im Sturm eroberten.

\section{Neuere Entwicklungen und Herausforderungen}

Nach dem Zweiten Weltkrieg entwickelte sich in der Deutschen Demokratischen Republik (DDR; 1949-1990) ein zentralistisches Gesundheitssystem, das der Prävention, der Gesundheitserziehung und dem Gesundheitsschutz in den Betrieben eine große Bedeutung zuwies. Die Gesundheitssysteme in der Bundesrepublik Deutschland (BRD) und der Schweiz wurden hingegen dezentral und libertär organisiert (s. a. Kap. 3). Krankheit berechtigte hier zur selbstverantwortlichen Inanspruchnahme von gesetzlich verankerten, versicherten medizinischen Leistungen. Der salutogenetische Public-Health-Ansatz (s. Kap. 1.3.1) rückte dabei in den Hintergrund. Krankheit wurde zunehmend als medizinisch-technisches Problem verstanden, für das Fachärzte und Krankenhäuser zuständig waren. Parallel zum Anstieg der Lebenserwartung sank die Kinderzahl pro Familie, ebenso der Anteil der Erwerbstätigen im Verhältnis zu den Nichterwerbstätigen.

Die demografische Entwicklung (s.a. Kap. 2.2) führte in den Industrienationen zu einer zunehmenden Alterung der Bevölkerung. Damit nahm auch die Häufigkeit chronisch-degenerativer Krankheiten, v. a. von Herz-Kreislauf-Erkrankungen und bösartigen Tumoren zu (s. Kap. 8.2 und Kap. 8.3). Gleichzeitig stieg die Anzahl der psychischen und psychosomatischen Erkrankungen (s. Kap. 8.8) an. Im Rahmen des Risikofaktorenmodells wurde nun nach biomedizinischen, aber auch nach psychosozialen Faktoren gesucht, die mit einer erhöhten Erkrankungswahrscheinlichkeit einhergehen. Man hoffte, hierdurch Strategien zur Prävention und Gesundheitsförderung entwickeln zu können (s. Kap. 4.1). Mit Hilfe der 1948 gestarteten FraminghamStudie, einer Kohortenstudie (s. a. Kap. 2.1.5) der Bevölkerung der Stadt Framingham im US-Bundesstaat Massachusetts, wurden z. B. verschiedene Risikofaktoren identifiziert, die zur Entstehung von Herzinfarkt und Schlaganfall beitragen (s. a. Kap. 8.2). In der Folgezeit wurden die Gesundheitswissenschaften zunehmend interdisziplinär und multiprofessionell (s. Kap. 1.4). Man wandte sich nun auch neuen Feldern zu, wie etwa der Evaluation und der Kosten-Nutzen-Bewertung medizinischer Maßnahmen (klinische Epidemiologie und Gesundheitsökonomie, s. a. Kap. 2.1.7 und Kap. 2.5), der 
Versorgung der Bevölkerung und der Steuerung der Gesundheitssysteme (Versorgungs- und Gesundheitssystemforschung, s. a. Kap. 3) und den Herausforderungen auf globaler Ebene (Global Health, s. a. Kap. 10).

Die Alterung der Bevölkerung (s. Kap. 2.2), die Zunahme von Übergewicht und chronisch-degenerativen Erkrankungen (s. a. Kap. 8) sowie die sozialen Ungleichheiten in Gesundheitszustand und Versorgung (s. a. Kap. 1.3.1 und Kap. 3) sind wichtige Felder, auf denen Public Health schon heute stark gefordert ist. Darüber hinaus gibt es noch einige Hindernisse, die es in den kommenden Jahren zu überwinden gilt. Hierzu gehören die dominante Rolle der kurativen Medizin in den fragmentierten Gesundheitssystemen (s. a. Kap. 3), die oft lückenhafte Zusammenarbeit zwischen den Gesundheitswissenschaften und den Einrichtungen des öffentlichen Gesundheitsdienstes sowie die fehlende Ausrichtung der Forschung auf die konkreten Fragestellungen der öffentlichen Gesundheitspolitik.

\subsection{Zentrale Konzepte und Themen}

\subsubsection{Gesundheit und Krankheit}

Ein Mensch ist nicht einfach entweder krank oder gesund. Die Betrachtungsweise kann sich schon durch die eingenommene Perspektive ändern: Eine Ärztin diagnostiziert bei einem Menschen eine Vorstufe von Krebs, der Betroffene verspürt jedoch noch keine Symptome und fühlt sich gesund. Auch handelt es sich bei Gesundheit und Krankheit nicht um ein Phänomen, das nur zwei Zustände einnehmen kann. Zwischen „krank“ und „gesund“ können zahlreiche Zwischenstufen bestehen. Zudem gibt es sehr unterschiedliche Vorstellungen darüber, wie Krankheit und Gesundheit entstehen. Krankheit und Gesundheit lassen sich darüber hinaus auch auf unterschiedlichen Ebenen betrachten, auf der des Individuums (dies ist v. a. die Sichtweise der Medizin) und auf der der Bevölkerung (dies entspricht der Perspektive von Public Health). Public Health und Medizin unterscheiden sich damit jedoch nicht nur in ihren Sichtweisen, sondern auch in den von ihnen gewählten Strategien, um Gesundheit zu erhalten, zu verbessern oder wiederherzustellen.

Es gibt zahlreiche Konzepte und Modelle, die z. T. sehr unterschiedliche Sichtweisen auf Krankheit und Gesundheit erlauben (siehe Internet-Ressourcen). Exemplarisch werden hier die Konzepte der Pathogenese und der Salutogenese vorgestellt.

\section{Pathogenese}

Mit dem Begriff der „Pathogenese“ bezeichnet man die Entstehung und Entwicklung einer Krankheit. Pathogenetische Konzepte beschäftigen sich mit Prozessen, die zu Krankheiten führen und untersuchen mögliche Risikofaktoren für die Entstehung 
von Krankheiten. Sie schauen dabei in erster Linie auf Veränderungen, die sich an Organen, Geweben und Zellen zeigen. Pathogenetische Konzepte bilden die Grundlage der naturwissenschaftlichen Medizin. Man kann sie auch als „Krankheitsmodelle“ verstehen und damit den weiter unten beschriebenen „Gesundheitsmodellen“ gegenüberstellen, derer sich Public Health häufig bedient.

Das biomedizinische Krankheitsmodell ist stark pathogenetisch geprägt. Es interpretiert Krankheit als eine Abweichung vom Normalzustand des Körpers. Krankheiten haben hier spezifische Ursachen (z. B. Bakterien). ÄrztInnen identifizieren diese Ursachen und können dann eine kausale ${ }^{2}$ - anstatt einer symptomatischen ${ }^{3}-$ Behandlung durchführen. Dieses stark naturwissenschaftlich beeinflusste Krankheitsmodell hat sich bei vielen Erkrankungen als sehr erfolgreich erwiesen. Daher werden u. a. in Deutschland und der Schweiz erhebliche Ressourcen in die Weiterentwicklung der Biomedizin investiert. Ein Schwerpunkt ist derzeit z. B. die Genomik (s. Kap. 1.7). In den vergangenen Jahrzehnten hat sich aber auch gezeigt, dass das biomedizinische Krankheitsmodell erhebliche Defizite aufweist. So geht es nicht ausreichend auf das individuelle Verhalten der Menschen ein, das insbesondere bei der Entstehung der immer bedeutsamer werdenden chronischen, nichtübertragbaren Krankheiten eine große Rolle spielt. Dieser Mangel wird durch das - ebenfalls stark pathogenetisch geprägte - Risikofaktorenmodell zumindest ansatzweise behoben (s. Kap. 2.1.1). Darüber hinaus bleiben gesellschaftliche Determinanten von Gesundheit und Krankheit nahezu unberücksichtigt. Dies ist in hohem Maße unbefriedigend, da u. a. sozioökonomische Benachteiligungen bei der Entstehung von Krankheit unzweifelhaft eine bedeutende Rolle spielen (s. Kap. 1.3.2). Weiterhin vermag das biomedizinische Krankheitsmodell nicht zu erklären, warum bestimmte Menschen gesund bleiben und wie sich Gesundheit fördern lässt. Spätestens hier zeigen sich die Stärken eines salutogenetischen Modells.

\section{Salutogenese}

Das Wort „Salutogenese“ bezeichnet analog dem Wort „Pathogenese“ die Entwicklung und Entstehung von Gesundheit. Es wurde in den 1970er-Jahren von Aaron Antonovsky im Rahmen seines salutogenetischen Modells geprägt (Näheres zu Antonovsky in den Internetquellen auf unserer Lehrbuch-Homepage). Anders als im medizinischen Denken steht hierbei die Gesundheit und nicht die Krankheit im Mittelpunkt. Antonovsky unterscheidet nicht zwischen zwei sich ausschließenden Begriffen „gesund“ und „krank“. Vielmehr interpretiert er Gesundheit und Krankheit als Endpunkte einer Linie. Zwischen diesen Endpunkten liegt ein Kontinuum von zahlreichen möglichen Zwischenstufen. Im Laufe des Lebens verändert sich der Gesundheitszustand eines

2 kausal: ursächlich

3 symptomatisch: an den Symptomen orientiert 
Menschen auf diesem Kontinuum ständig. Antonovsky betrachtet Krankheiten somit als einen normalen Teil des menschlichen Lebens. Gesundheit ist also nicht der Regelfall und Krankheit nicht lediglich eine Abweichung von einem normalerweise bestehenden Gleichgewicht (Homöostase). Antonovsky spricht in seinem Modell der Salutogenese von einem Zustand der Heterostase, in dem sich der Mensch befindet. Er betont damit die ständigen Veränderungen, denen der Organismus infolge der Einwirkung äußerer Stressoren ausgesetzt ist. Nur durch ständige aktive Anpassungsleistungen und Auseinandersetzungen mit solchen Stressoren bleiben Menschen gesund.

Die beiden äußeren Punkte des von ihm beschriebenen Kontinuums bezeichnet Antonovsky als „health-ease“ (Gesundheit) und „dis-ease“ (Krankheit). Hiervon leitet er die Bezeichnung HEDE-Kontinuum ab. Aus salutogenetischer Sicht soll ein Mensch stets aktiv danach streben, auf diesem Kontinuum möglichst nahe an den Punkt „Gesundheit“ zu gelangen. Widerstandsressourcen helfen ihm dabei, Stressoren zu überwinden und sich somit auf dem HEDE-Kontinuum in Richtung Gesundheit zu bewegen. Solche Ressourcen können zum einen auf der gesellschaftlichen Ebene liegen (hierzu gehört z.B. ein intaktes gesellschaftliches Umfeld). Zum anderen verfügt jeder Mensch aber auch in unterschiedlichem Ausmaß über individuelle Ressourcen (s. Kap. 4.1), etwa bei der Problemlösefähigkeit ( $\rightarrow$ Kognition), beim Selbstvertrauen ( $\rightarrow$ Psyche), bei der durch Training erworbene Ausdauer ( $\rightarrow$ Körper) oder in finanzieller Hinsicht ( $\rightarrow$ Ökonomie).

Hat ein Mensch belastende Situationen wiederholt erfolgreich bewältigt, kann sich bei ihm ein zunehmendes Kohärenzgefühl einstellen (auch im Deutschen wird hierfür häufig der englische Begriff Sense Of Coherence, SOC, benutzt). Menschen mit einem ausgeprägten Kohärenzgefühl sind dadurch in der Lage, mit Stressoren erfolgreich umzugehen oder diese sogar als positive Herausforderung $\mathrm{zu}$ erleben. Angemessene Bewältigungsstrategien (Coping-Strategien; s. Kap. 4.2.1) wirken sich in Verbindung mit einem starken Kohärenzgefühl förderlich auf die Gesundheit aus. Viele Public-Health-Strategien zur Förderung der Gesundheit in der Bevölkerung zielen daher darauf $\mathrm{ab}$, gesellschaftliche und individuelle Ressourcen $\mathrm{zu}$ stärken. Dieser Ansatz unterscheidet sich damit substanziell von der Betrachtungsweise der Medizin, die einen pathogenetischen Ansatz vertritt.

\subsubsection{Gesundheitliche Ungleichheiten}

Eines der Kernthemen von Public Health ist die Ungleichheiten zwischen verschiedenen Bevölkerungsgruppen in Bezug auf ihre Gesundheit. Die vorhandenen Unterschiede im Hinblick auf soziale Schicht, Region, Ethnie, Nationalität, Alter und Geschlecht gehen oft mit gesundheitlichen Ungleichheiten einher. Diese Ungleichheiten betreffen neben dem Gesundheitszustand und den Gesundheitschancen (s. a. Kap. 4.1) auch das Gesundheitsverhalten und den Lebensstil (s. a. Kap. 4.2.3) sowie den Zugang und die Inanspruchnahme von Leistungen des Gesundheitssys- 
tems (s.a. Kap. 3). Sie sind in der Regel nicht durch unterschiedliche Bedürfnisse der Menschen gerechtfertigt, sondern entstehen aufgrund von Privilegien oder Benachteiligungen. In diesem Abschnitt diskutieren wir stellvertretend hierfür die Ungleichheiten, die im Zusammenhang mit der sozialen Schichtzugehörigkeit und dem Geschlecht auftreten. Selbstverständlich bestehen oft gleichzeitig auch andere Formen der gesundheitlichen Ungleichheit, z. B. infolge des Alters oder der ethnischen Zugehörigkeit.

\section{Soziale Ungleichheit und Gesellschaft}

Sowohl in reichen wie auch in armen Ländern gibt es innerhalb von Gesellschaften oft große Unterschiede zwischen den Menschen hinsichtlich bestimmter Merkmale wie Einkommen, beruflicher Position, Bildung und Sozialprestige. Diese Merkmale bilden die Grundlage für die Eingruppierung der Menschen in soziale Schichten. Entsprechend der unterschiedlichen Ausstattung haben die Menschen in den verschiedenen sozialen Schichten unterschiedliche Chancen, nicht nur in gesellschaftlicher Hinsicht, sondern - damit einhergehend - auch bezüglich ihrer Gesundheit. Es sind also nicht nur Krankheitserreger oder individuelle gesundheitsschädigende Verhaltensweisen, die zu Erkrankungen führen. Vielmehr trägt auch die Ungleichverteilung von Ressourcen zu einem höheren Krankheitsrisiko in den benachteiligten Schichten bei.

Ungleichheit und Ungerechtigkeit: Das Wort „Ungleichheit“ (Inequality) bezeichnet zunächst nur Unterschiede in den gesundheitlichen Chancen von Bevölkerungsgruppen. Solche Unterschiede kommen häufig vor. Oftmals sind sie nicht zu ändern oder werden sogar freiwillig von den Betroffenen hervorgerufen. So haben z. B. ältere Menschen ein höheres Risiko zu versterben als jüngere - daran ist leider nichts zu ändern. Ein anderes Beispiel sind Mountainbiker, die ein höheres Verletzungsrisiko als Menschen haben, die keinen Sport treiben. Dieses zusätzliche Risiko gehen sie aber freiwillig ein. Gleichzeitig ziehen sie möglicherweise auch gesundheitliche Vorteile aus dieser Tätigkeit.

Zahlreiche sozial bedingte gesundheitliche Unterschiede zwischen den Bevölkerungsgruppen sind jedoch vermeidbar und vor allem so gravierend, dass sie nicht einfach hingenommen werden können. Man spricht dann von Ungerechtigkeit (Inequity). So sind MigrantInnen in Deutschland eine sozial benachteiligte Gruppe. Sie weisen eine zwei- bis dreimal so hohe Säuglingssterblichkeit (Todesfälle im ersten Lebensjahr pro 1.000 Lebendgeborene) wie die nicht migrierte Mehrheitsbevölkerung auf (s. a. Kap. 10.2.7). Todesfälle bei Säuglingen sind schwerwiegende Vorfälle, die vielfach vermeidbar sind. Um sie zu verhindern, müssen Schwangerenvorsorge, Geburtshilfe und kinderärztliche Versorgung für alle in gleicher Weise zugänglich sein und in gleich hoher Qualität angeboten werden. Menschen mit geringerer Bildung oder mit Problemen mit der deutschen Sprache nehmen jedoch Leistungen oft zu spät in Anspruch oder seltener als der Durchschnitt der Bevölkerung. Der Zugang zu Gesundheitseinrichtungen ist damit von der sozialen Lage abhängig, in der sich ein 
Mensch befindet. Wenn also Unterschiede in der Säuglingssterblichkeit zwischen den sozialen Schichten auftreten, so handelt es sich dabei um eine gesundheitliche Ungerechtigkeit.

Solche gesundheitlichen Ungerechtigkeiten treten oft in noch stärkerem Maße zwischen armen und reichen Ländern auf. Tabelle 10.2 in Kap. 10 zeigt dies eindrücklich am Beispiel der Säuglingssterblichkeit. Sie ist in Malawi 14,5-mal so hoch wie in der Schweiz, in Österreich oder in Deutschland. Die Gründe für diese Unterschiede sind nahe liegend. Malawi ist ein Land mit einem sehr niedrigem Entwicklungsstand (Least Developed Country, siehe Tab. 10.1) und sehr niedrigem pro-Kopf-Einkommen, während die Schweiz, Österreich und Deutschland zu den High-income-Ländern gehören.

Die Whitehall-Studie: Die soziale und wirtschaftliche Lage von Menschen nimmt also großen Einfluss auf ihre Gesundheitschancen. Diese Erkenntnis gilt in den Industrienationen in ähnlicher Weise auch für chronische Erkrankungen (z. B. für Herz-Kreislauf-Krankheiten). Dies haben sozialepidemiologische Studien seit den 1960er-Jahren eindrücklich belegt. Am bekanntesten ist hier die Whitehall-Studie, die 1967 startete und nach dem Regierungsgebäude Whitehall in London benannt wurde. An dieser Kohortenstudie (s. Kap. 2.1) nahmen 18.000 männliche Angestellte der britischen Regierung teil. Die Studienergebnisse zeigten, dass Männer, die in der niedrigsten Job-Kategorie arbeiteten, eine höhere Sterblichkeit im Hinblick auf nahezu alle wichtigen Todesursachen hatten als Männer in der höchsten Job-Kategorie. Dies lag nicht etwa nur daran, dass schlechter gestellte Menschen meist auch ungesünder leben. Der Gradient blieb auch dann bestehen, wenn man die Unterschiede in der Prävalenz (Häufigkeit) von Risikofaktoren wie Rauchen statistisch ausglich (adjustierte). Zugespitzt formuliert: Pförtner haben hiernach ein höheres Risiko, einen Herzinfarkt zu erleiden oder frühzeitig zu versterben als leitende Angestellte. Der Herzinfarkt ist damit nicht eine Krankheit der Manager und Chefs, sondern der Gemanagten - der Menschen, die ein geringes Einkommen, einen niedrigen Bildungsstand und geringe Gestaltungsmöglichkeiten in ihrem Leben haben. Mittlerweile liegen aus Deutschland und der Schweiz ähnliche Beobachtungen vor. Abb. 1.4 aus der Schweiz zeigt die weitere Lebenserwartung von Männern verschiedener Altersgruppen und unterschiedlicher Bildung. Als Vergleichsgruppe dienen Männer, die lediglich die obligatorische Schulbildung durchlaufen haben. In allen Altersgruppen haben die Männer eine umso höhere weitere Lebenserwartung, je höher ihr Bildungsgrad ist.

Seit 1985 wird eine ähnliche Kohortenstudie wie die oben beschriebene WhitehallStudie unter dem Namen Whitehall II durchgeführt, nun auch mit weiblichen Teilnehmern. Sie versucht zu klären, wie der gesundheitliche Gradient in Abhängigkeit von der beruflichen Stellung oder der sozialen Lage entsteht. Die Ergebnisse legen nahe, dass Stress hierbei eine große Rolle spielt und dass es verschiedene Arten von Stress gibt. Zur gesundheitlichen Ungleichheit trägt v. a. eine Form des Stresses bei, die entsteht, wenn den Betroffenen die Möglichkeit fehlt, ihr Leben oder ihre Arbeit selbst zu 


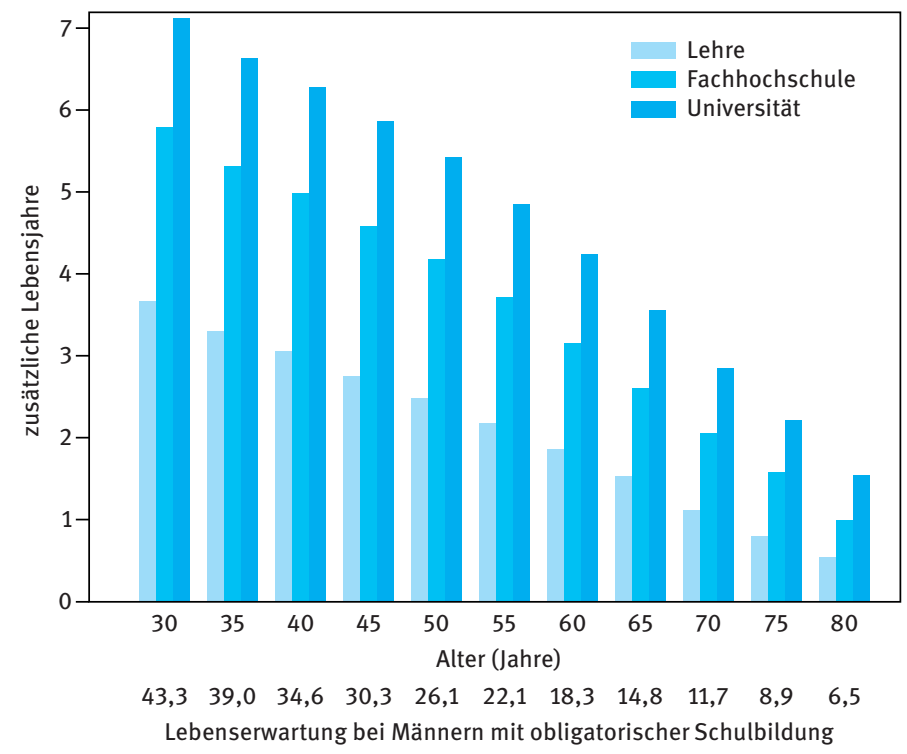

Abb. 1.4: Die weitere Lebenserwartung bei Männern in der Schweiz in Abhängigkeit von Alter und Bildung. Die Zahlen der unteren Zeile geben die Lebenserwartung bei Männern mit obligatorischer Schulbildung (Primarschule) an. Die Balken darüber zeigen die zusätzliche Anzahl an Lebensjahren bei Männern mit einer Berufsbildung in einer Firma (Lehre) und bei Männern mit einem Diplom einer Fachhochschule bzw. Universität. (Quelle: Spörri et al. Swiss Medical Weekly, 2006).

gestalten (s. a. Kap. 4.2.2, Kap. 7.3.1 und Kap. 8.2). Mittlerweile haben weitere Studien auch andere wichtige Faktoren identifiziert. So haben sozial schlechter gestellte Menschen oft einen schlechteren Zugang zu den Gesundheitsdiensten - dies gilt beispielsweise für MigrantInnen in Deutschland (s. o. und Kap. 10.2.7). Auch gibt es Hinweise darauf, dass bereits während der Schwangerschaft und in der frühen Kindheit Krankheitsrisiken „programmiert“ werden (vgl. Kap. 1.7 und Kap. 5.1). Wer unter ungünstigen Bedingungen aufwächst, hätte demnach im späteren Leben ein höheres Erkrankungsrisiko als Menschen, die in wirtschaftlich entspannten Verhältnissen zur Welt kommen. Natürlich spielt hier auch eine Rolle, dass gesundheitsschädliche Verhaltensweisen unter sozial benachteiligten Menschen häufiger vorkommen. Darüber hinaus können Menschen, die wegen ihrer sozialen Benachteiligung ein höheres Krankheitsrisiko haben, dadurch auch sozial weiter absteigen, was wiederum das Krankheitsrisiko erhöht - ein Teufelskreis aus Krankheit und Armut.

Interventionen zur Verbesserung der Gesundheit auf der Bevölkerungsebene: Die heute vorliegenden Erkenntnisse aus der Sozialepidemiologie bestätigten nachdrücklich, dass es nicht nur individuelle, medizinische Risikofaktoren gibt, die unsere 
Gesundheit beeinflussen, sondern auch gesellschaftlich bedingte krankmachende oder schützende Faktoren - auch in der Schweiz, in Österreich und in Deutschland. Um die Gesundheit der Bevölkerung zu verbessern, reichen daher medizinische Maßnahmen allein nicht aus. Vielmehr ist es erforderlich, die Lebensbedingungen benachteiligter Gruppen zu verbessern. Auch müssen diese Gruppen gezielt angesprochen werden, um ihr gesundheitliches Wissen und ihren Zugang zu Gesundheitsdiensten zu verbessern. Die AutorInnen eines Berichts der Weltgesundheitsorganisation WHO, der unter dem Namen The Social Determinants of Health (Die sozialen Determinanten von Gesundheit) im Jahr 2012 vorgelegt wurde, kommen zum Schluss, dass hierfür die Ungleichverteilung von gesellschaftlichen Ressourcen (v. a. der finanziellen Mittel und des politischen Einflusses) reduziert werden muss.

\section{Ungleichheit zwischen den Geschlechtern}

Das Geschlecht gehört zu den wichtigsten Determinanten gesundheitlicher Ungleichheiten. Im Englischen werden die kleineren und größeren Unterschiede zwischen Frauen und Männern vereinfachend in zwei Kategorien unterteilt:

- Der Begriff „Sex“ bezeichnet die biologischen (u. a. genetischen, anatomischen, physiologischen, immunologischen) Unterschiede zwischen den Geschlechtern. Das biologische Geschlecht beeinflusst z. B.

- die Wahrscheinlichkeit geboren zu werden: Das natürliche Verhältnis von Jungen zu Mädchen liegt hier bei 1,05 (105 Jungen : 100 Mädchen).

- das Risiko, an bestimmten Erkrankungen zu leiden: So kommen z. B. Autoimmunerkrankungen bei Frauen häufiger vor als bei Männern.

- die Ausprägung von bestimmten Symptomen: Die beim Herzinfarkt auftretenden Symptome unterscheiden sich z. B. in Abhängigkeit vom Geschlecht der Betroffenen.

- die Behandlungsresultate: Frauen bilden z. B. nach Impfungen weniger Antikörper.

- die Lebenserwartung: In den Industrienationen liegt sie bei Frauen um etwa 5 Jahre höher als bei Männern (s. a. Kap. 2.2.4).

- Im Gegensatz hierzu beschreibt der Begriff „Gender“ die psychologischen, sozialen und kulturellen Dimensionen des Geschlechts. Damit sind insbesondere die sozialen Rollen, Beziehungen, Verhaltensweisen und die Wertschätzung gemeint, die Frauen und Männern in einer Gesellschaft zugeschrieben werden. In Gesellschaften mit Bevorzugung männlicher Nachkommen, z. B. in Indien oder China, leistet die pränatale Bestimmung des Geschlechts mit Hilfe von Ultraschalluntersuchungen der selektiven Abtreibung von weiblichen Embryonen Vorschub. Obwohl es in Indien verboten ist, den Eltern das Geschlecht ihres Kindes während der Schwangerschaft mitzuteilen, wurden bei der Volkszählung im Jahre 2011 in Indien pro 1.000 Jungen unter sechs Jahren nur noch 914 gleichaltrige Mädchen registriert (Verhältnis 1,09). 
Ein Beispiel für die Gesundheitsrelevanz der Geschlechterrollen ist die alkoholassoziierte Sterblichkeit: Das Trinken von Alkohol in großen Mengen ist in vielen Ländern Teil des männlichen Rollenverständnisses. So ist in Russland das „Zapoi“, ein mehrtägiger Alkoholexzess, ein lebensgefährliches Ritual unter den Männern (s. Box 10.1.2 in Kap. 10). Es erstaunt deshalb nicht, dass Alkohol weltweit bei Männern für einen deutlich höheren Anteil an verlorenen Lebensjahren (Disability Adjusted Life Years) verantwortlich ist als bei Frauen (s. a. Kap. 10.1).

Die Förderung der Chancengleichheit von Männern und Frauen ist ein wichtiges Ziel der Politik. Sie gehört darüber hinaus zu den Entwicklungszielen der Vereinten Nationen (Sustainable Development Goals [SDGs], s. a. Kap. 10.3.1). Der Europarat hat in diesem Zusammenhang den Begriff „Gender Mainstreaming“ für eine gleichstellungsorientierte Politik auf allen Ebenen geprägt. Für Public Health und die Gesundheitspolitik bedeutet dies, dass die gesundheitliche Situation von Frauen und Männern in allen Bereichen der öffentlichen Gesundheit berücksichtigt werden soll. Auch die Versorgung soll verstärkt auf die spezifischen Bedürfnisse von Frauen und Männern ausgerichtet werden. Weiterhin sollen Public-Health-Maßnahmen und -Programme bezüglich ihrer Auswirkungen auf Geschlechterungleichheiten überprüft werden, und auch in der Gesundheitsberichterstattung soll die Geschlechterperspektive Berücksichtigung finden. Dies alles soll jedoch nicht isoliert geschehen, sondern unter Berücksichtigung anderer Dimensionen von Ungleichheit - wie z. B. von Ungleichheit, die durch die sozio-ökonomische Lage, das Alter oder den Migrationshintergrund hervorgerufen wird.

\subsection{Die Disziplinen der Public Health}

Unter dem Dach von Public Health wirken Disziplinen zusammen, die aus zwei unterschiedlichen wissenschaftlichen Traditionen kommen, der medizinisch-naturwissenschaftlichen und der sozial- und verhaltenswissenschaftlichen Tradition. Methodische Kernbereiche des interdisziplinären Fachs Public Health sind die Epidemiologie (s. Kap. 2.1) - ergänzt durch die Demografie (Kap. 2.2) und die Biostatistik (Kap. 2.3) - sowie die Sozialwissenschaften (s. Kap. 2.4) und die Gesundheitsökonomie (s. Kap. 2.5). Während die Epidemiologie quantitativ arbeitet, kommen in den Sozialwissenschaften sowohl quantitative als auch qualitative Methoden und Instrumente zum Einsatz.

Wichtige Einzeldisziplinen von Public Health sind:

- Sozialmedizin: Hierunter versteht man den Bereich der Medizin, der Zusammenhänge zwischen gesellschaftlichen Faktoren wie Einkommen oder Berufstätigkeit und gesundheitlichen Outcomes wie Erkrankung oder Tod untersucht (s.u.a. Kap. 4.2.2 und Kap. 4.2.3). Es bestehen enge Beziehungen zur Arbeitsmedizin (s. Kap. 7), deren Ziel es ist, arbeitsbedingte Erkrankungen u. a. durch Vorsorgemaßnahmen zu verhindern oder diese abzumildern. 
- Medizinsoziologie: Sie wendet die Theorien und Methoden der Soziologie an, um Gesundheit und Krankheit $\mathrm{zu}$ beschreiben und zu erklären. Im Zentrum stehen dabei die sozialen Bedingungen, Ursachen und Konsequenzen von Gesundheit und Krankheit. Die Medizinsoziologie analysiert die Einflüsse sozialer Organisation und Institutionen auf die Bevölkerungsgesundheit genauso wie die Zusammenhänge von sozialen Bedingungen, individuellem Verhalten und Erkrankungsrisiko. Ihre Erkenntnisse fließen in Maßnahmen der Prävention und der Gesundheitsförderung (s. Kap. 4.1) ein.

- Umweltmedizin: Die Umweltmedizin beschäftigt sich mit den Einflüssen von Umweltnoxen wie Lärm oder Luftschadstoffen auf die Gesundheit der Bevölkerung (s. Kap. 6).

- Präventivmedizin: Sie befasst sich mit der Krankheitsvorsorge und der Verhütung von Krankheiten, u. a. durch Prävention oder Früherkennung. Beispiele hierfür sind Impfungen zur Kontrolle von Infektionskrankheiten (Communicable Diseases; s. Kap. 9) und Screening-Programme zur Früherkennung von chronischen Krankheiten wie Herz-Kreislauf- oder Krebserkrankungen (Non Communicable Diseases; s. Kap. 8 sowie Kap. 4.4 und Kap. 4.5).

- Gesundheitspsychologie und -pädagogik: Diese Disziplinen untersuchen das menschliche Erleben und Verhalten bzw. deren Veränderungen. Ihre Erkenntnisse werden u. a. im Bereich der Gesundheitsförderung angewandt (s. Kap. 4.1.2 und Kap. 4.2.2).

- Gesundheitspolitik: Sie beschäftigt sich damit, wie Institutionen im Gesundheitssystem entstehen und wie diese arbeiten, wie dort Prozesse ablaufen und Entscheidungen getroffen werden (s. z. B. Kap. 3 und Kap. 4.1.3).

- Gesundheitsökonomie. Die Gesundheitsökonomie (s. Kap. 2.5) untersucht alle wirtschaftlichen Aspekte von Gesundheit, Krankheit und Gesundheitsversorgung.

- Organisations- und Managementwissenschaften: Sie befassen sich mit Prozessabläufen und Entscheidungsfindungen innerhalb von Institutionen, wie z.B. von Krankenhäusern.

- Ethik: Die Ethik beschäftigt sich mit gutem, richtigem und gerechtem menschlichem Handeln - ein Aspekt, der z. B. bei der Verteilung knapper Ressourcen eine große Rolle spielt (s. Kap. 1.6).

Die hier aufgelisteten Einzeldisziplinen lassen sich hinsichtlich ihres jeweiligen Forschungsgegenstands nicht immer eindeutig voneinander trennen. Deutlich wird das in den Bereichen Sozialmedizin und Medizinsoziologie. Dort wird oft die gleiche Thematik untersucht, jedoch aus unterschiedlichen wissenschaftlichen Perspektiven. Andererseits gibt es Public-Health-Forschungsfelder, die meist nicht als eigene Disziplinen ausgewiesen werden, die aber die interdisziplinäre Arbeitsweise von Public Health sehr deutlich aufzeigen. Ein Beispiel hierfür ist die Gesundheitssystem- und Versorgungsforschung. Sie untersucht die Struktur, Leistungsfähigkeit und Wirksam- 
keit von Angeboten in den Gesundheitssystemen. Erreichbarkeit, Zugang und Nutzen von Angeboten sowie deren Kosten-Nutzen-Verhältnis spielen dabei eine bedeutende Rolle. Hierzu bedient sie sich u. a. verschiedener Methoden und Ansätze der Epidemiologie, der empirischen Sozialforschung, der Gesundheitspolitik, der Management- und Organisationsforschung sowie der Ethik.

Die vorliegende Liste kann keinen Anspruch auf Vollständigkeit erheben. So lässt sich auch argumentieren, dass weitere Fächer wie z. B. die Sportmedizin zu Public Health gehören, da sie sich im Bereich der Prävention und Gesundheitsförderung (oft in Zusammenarbeit mit der Gesundheitspsychologie und -pädagogik oder der Präventivmedizin) engagieren. Andererseits führen nicht alle Versuche einer Zusammenarbeit zwischen medizinisch-naturwissenschaftlichen Fächern und Public Health stets zu Ergebnissen, die aus Public-Health-Sicht unmittelbar relevant sind. Kapitel 1.7 diskutiert dies am Beispiel der Public Health Genomics.

\subsection{Ansatzpunkte der Prävention}

Prävention bedeutet im wörtlichen Sinne, einer Krankheit „zuvorzukommen“ (von lat. praevenire). Um dies zu erreichen, kann Prävention an verschiedenen Punkten ansetzen:

- $\quad$ auf dem Weg von Gesundheit über Krankheit zum Tod: Primär-, Sekundär- und Tertiärprävention

- auf der Bevölkerungsebene oder bei Risikogruppen: Bevölkerungs- oder Hochrisikostrategie

- am Individuum oder seiner Umwelt: Verhaltens- und Verhältnisprävention (s. Kap. 4.1)

Ziel von Prävention ist die Verbesserung der Gesundheit der Bevölkerung insgesamt, aber auch der Gesundheit von Bevölkerungsgruppen oder einzelnen Personen. In den folgenden Abschnitten erläutern wir hierzu einige Konzepte und Begriffe.

\subsubsection{Primär-, Sekundär- und Tertiärprävention}

Leider werden die folgenden Begriffe nicht immer einheitlich genutzt. Zudem gibt es Übergänge zwischen den beschriebenen Formen.

Primärprävention hat zum Ziel, das Auftreten von Gesundheitsschäden, Neuerkrankungen und Todesfällen in der Bevölkerung zu vermeiden oder zumindest die Wahrscheinlichkeit zu senken, dass die betreffenden Schädigungen oder Krankheiten auftreten. Klassische Beispiele für Primärprävention sind Maßnahmen zum Nichtraucherschutz (z.B. durch Rauchverbote in Gaststätten und öffentlichen Räumen) oder zum Anheben des „Einstiegsalters“ beim Rauchen (z. B. durch die Besteuerung 
von Tabakprodukten, um eine finanzielle Barriere zu errichten). Auch Impfungen, wie etwa die Masernimpfung bei Kindern, gehören zur Primärprävention.

Sekundärprävention zielt darauf ab, klinisch noch unauffällige Frühformen von Erkrankungen zu erkennen und dadurch rechtzeitig zu behandeln, sodass die Erkrankung nicht fortschreitet oder sogar geheilt werden kann. Ein klassisches Beispiel hierfür ist das bevölkerungsweite Screening zur Früherkennung von bestimmten Krankheiten wie Brust- oder Darmkrebs (s. Kap. 4.5).

Ziel der Tertiärprävention ist es, die Verschlimmerung einer bereits manifesten Erkrankung zu verhindern oder den Vorgang zu verlangsamen. Weitere mögliche Ziele sind die Verbesserung der Lebensqualität oder der sozialen Funktionsfähigkeit. Ein typisches Beispiel hierfür sind Rehabilitationsmaßnahmen nach Eintritt einer schweren Herz-Kreislauf- oder Krebserkrankung.

Einige präventive Maßnahmen können jedoch auch Aspekte von Primär-, Sekundär- und Tertiärprävention beinhalten. So lassen sich beispielsweise viele Maßnahmen der Gesundheitsberatung mehr als einem der drei genannten Ansatzpunkte zuordnen. Eine Ernährungsberatung kann bei gesunden Menschen das Ziel haben, das Auftreten eines Diabetes mellitus Typ 2 von vornherein zu verhindern. Bei Menschen mit mäßig erhöhtem Blutzuckerspiegel soll durch Ernährungsberatung das Auftreten einer klinischen Symptomatik vermieden werden. Schließlich soll sie bei manifesten DiabetikerInnen helfen, das Risiko von Komplikationen zu senken.

Es gibt unterschiedliche Meinungen darüber, ob kurative medizinische Maßnahmen auch als Sekundär- oder Tertiärprävention bezeichnet werden können. Ein Beispiel dafür ist die Gabe von antiretroviralen Medikamenten bei HIV-positiven Menschen. Sind bei den Betroffenen schon Symptome aufgetreten, kann die Medikation dazu dienen, eine Verschlimmerung der Erkrankung sowie das Auftreten von durch die Immunschwäche bedingten Folgeerkrankungen zu verhindern. Die Gabe antiretroviraler Medikamente könnte hier somit als Tertiärprävention angesehen werden. Bei klinisch noch unauffälligen Menschen mit positivem HIV-Test kann die Medikation das Auftreten von Symptomen verhindern. Hier könnte die Maßnahme also als Sekundärprävention interpretiert werden. Da eine antiretrovirale Therapie die Viruslast im Blut der Behandelten erheblich senken kann, sodass sich die Ansteckungsgefahr für ihre Sexualpartner verringert, handelt es sich auch um eine primärpräventive Maßnahme.

\subsubsection{Bevölkerungs- und Hochrisikostrategie}

Die Konzepte der Hochrisikostrategie und der Bevölkerungsstrategie wurden in den 1980er Jahren vom britischen Epidemiologen Geoffrey Rose (1926-1993) im Zusammenhang mit der Primärprävention kardiovaskulärer Erkrankungen (s.a. Kap. 8.2) entwickelt. Diese Konzepte sind weiterhin von großer Bedeutung. 


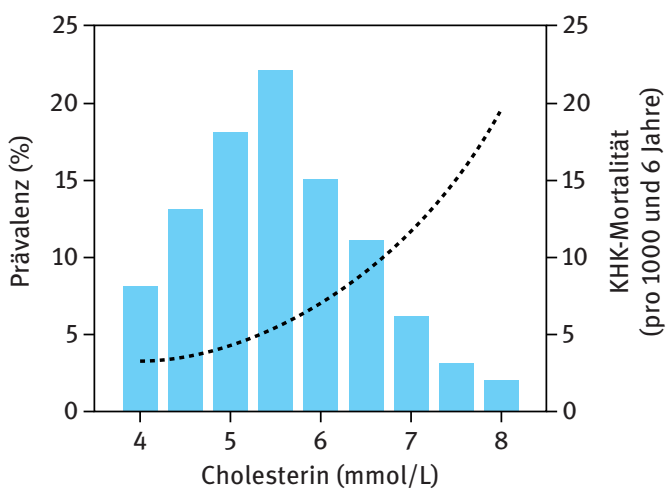

Durch erhöhtes Cholesterin verursachte KHK-Todesfälle (\%):

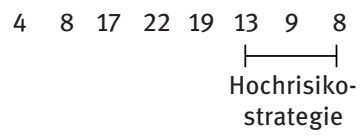

Abb. 1.5: Verteilung der Serumcholesterinwerte bei Männern in Korrelation zum Risiko, an einer koronaren Herzkrankheit (KHK) zu sterben [Säulen] sowie Anzahl der KHK-Todesfälle pro 1.000 Männer innerhalb von 6 Jahren im Verhältnis zu ihren Cholesterinwerten [gestrichelte Linie]. Die Hochrisikostrategie zielt auf eine Reduktion des Risikos von Männern mit deutlich erhöhten Werten. Unter den Verstorbenen sind aber wesentlich mehr Männer mit mäßig als mit stark erhöhten Cholesterinwerten.

(Quelle: modifiziert nach Rose G. The Strategy of Preventive Medicine. Oxford University Press, 1992).

Die Hochrisikostrategie ist eine logische Fortsetzung des Denkens in Kategorien, das die klinische Medizin prägt. Menschen sind entweder gesund oder krank und benötigen eine Therapie oder nicht. Analog hierzu zielt die Hochrisikostrategie darauf ab, die Menschen, die ein erhöhtes Risiko haben, in Zukunft eine Erkrankung zu entwickeln, zu identifizieren, um dieses Risiko durch geeignete Maßnahmen zu verringern. Abb. 1.5 illustriert dies anhand des Gesamtcholesterinwertes im Blut und der Sterblichkeit durch die koronare Herzkrankheit (KHK). Die Daten stammen aus einer großen Kohortenstudie, an der in den USA mehr als 350.000 Männer teilnahmen. Sie wurden im Rahmen des Multiple Risk Factor Intervention Trial untersucht und nachverfolgt. Die Abbildung zeigt die Verteilung der Cholesterinwerte in der Bevölkerung (Säulen) und den Anstieg der KHK-Mortalität in Korrelation zu den Cholesterinwerten (unterbrochene Linie). Bei den meisten Männern wurden Cholesterinwerte zwischen 4,5 mmol/1 und 6,0 mmol/1 gemessen. Die in einem Zeitraum von sechs Jahren gemessene KHK-Mortalität lag in Abhängigkeit von den Cholesterinwerten der Teilnehmer zwischen ca. 3 pro 1.000 und 20 pro 1.000 Teilnehmer. In der Hochrisikogruppe (Männer mit sehr hohen Cholesterinwerten) verstarb somit einer von 50 Männern an einer KHK. Allerdings waren in dieser Studie nur 2\% der teilnehmenden Männer betroffen. Die Zahlen unterhalb der Grafik geben den Prozentsatz der Todesfälle an, der in jeder Cholesterinkategorie durch die erhöhten Werte verursacht wurde. Diese Zahlen illustrieren eindrücklich die Grenzen 


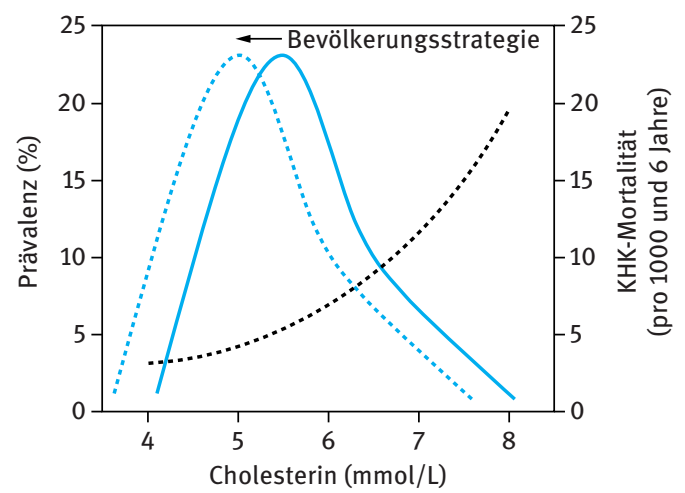

Abb. 1.6: Verteilungen der Serumcholesterinwerte [grüne durchgezogene Linie] in der männlichen Bevölkerung und des auf den Cholesterinwert bezogenen Risikos bei Männern, an einer koronaren Herzkrankheit (KHK) zu sterben [schwarze gestrichelte Linie]. Die Populationsstrategie zielt auf die Verschiebung der gesamten Cholesterinverteilung in Richtung günstigerer Werte [grüne gestrichelte Linie] und reduziert deshalb das Risiko aller Männer, an einer KHK zu versterben.

(Quelle: modizifiert nach Rose G. The Strategy of Preventive Medicine. Oxford University Press, 1992).

der Hochrisikostrategie. Nur in 30\% der Todesfälle sind Männer betroffen, bei denen deutlich erhöhte Cholesterinwerte ( $\geq 7 \mathrm{mmol} / \mathrm{l}$ ) gemessen wurden. Die überwiegende Mehrzahl der Todesfälle ( $70 \%$ ) trat bei Männern mit moderat erhöhten Cholesterinwerten auf (Werte zwischen 5,5 und $7 \mathrm{mmol} / \mathrm{l}$ ).

Wird bei der Prävention ausschließlich eine Hochrisikostrategie eingesetzt, profitiert somit nur eine Minderheit von Menschen mit deutlich erhöhtem Risiko. Die Mortalität insgesamt wird nur wenig beeinflusst. Diese Situation trifft auch für viele andere kardiovaskuläre Risikofaktoren wie z. B. Blutdruck, Entzündungsmarker oder glykosyliertes HbA1c zu. Auch beim Verhältnis zwischen Augeninnendruck und dem Auftreten eines grünen Stars (Glaukom) oder der Knochendichte und dem Frakturrisiko ist die Situation ähnlich.

Die Bevölkerungsstrategie berücksichtigt im Gegensatz zur Hochrisikostrategie die Verteilung des Risikos in der gesamten Bevölkerung und strebt danach, diese Risikoverteilung hin zu günstigeren Werten zu verschieben (Abb. 1.6). Eine erfolgreiche Bevölkerungsstrategie sucht somit eine ähnliche Verteilung des Risikofaktors auf einem niedrigeren Niveau zu erreichen (gestrichelte grüne Linie in Abb. 1.6). Ihr Nutzen ergibt sich einerseits aus der Verschiebung von Hochrisikopersonen heraus aus der Gefahrenzone. Noch wichtiger im Hinblick auf den Bevölkerungsnutzen ist jedoch die insgesamt große Summe des für jeden Einzelnen relativ kleinen Nutzens bei sehr vielen Personen in der Mitte der Verteilung.

Es kann nun einfach berechnet werden, ob der Gesamtnutzen bei der Hochrisiko- oder der Bevölkerungsstrategie größer ausfällt. Eine Tabelle in Kap. 1 unserer 
Lehrbuch-Homepage zeigt ausführliche Berechnungen für die kardiovaskuläre ${ }^{4}$ Primärprävention bei britischen Männern mittleren Alters. Eine geringfügige Verschiebung der Blutdruckverteilung um 5\% in Richtung niedrigerer Werte hat z. B. einen größeren Effekt auf die Inzidenz von kardiovaskulären Ereignissen (16\% Reduktion im Rahmen einer Bevölkerungsstrategie) als die blutdrucksenkende Behandlung von all denjenigen Männern, die ein Zehnjahresrisiko von 20\% oder mehr aufweisen (11\% Reduktion im Rahmen einer Hochrisikostrategie). Ein Risiko von $20 \%$ für den Eintritt eines kardiovaskulären Ereignisses wird in den aktuellen Richtlinien allgemein als Schwellenwert für einen Therapiebeginn mit blutdrucksenkenden Mitteln verwendet. Erst wenn bereits ab einem Risiko von $15 \%$ blutdrucksenkende Medikamente gegeben werden, wird dieselbe Reduktion (16\%) erreicht. In dieser Situation müssten jedoch $50 \%$ aller Männer Medikamente einnehmen! Eine ähnliche Situation ergibt sich für die Cholesterinsenkung oder die kombinierte Reduktion von Cholesterinwerten und Blutdruck.

\subsubsection{Das Präventionsparadox}

Wie das Beispiel zeigt, kann der Bevölkerungsansatz durch eine kleine Reduktion des Risikos bei allen Menschen eine beträchtliche Reduktion der Inzidenz (s. Kap. 2.1.2) erreichen. Allerdings ist es für den Einzelnen sehr unwahrscheinlich, dass er oder sie persönlich von dieser Maßnahme profitieren wird. In diesem Zusammenhang prägte Rose 1981 den Begriff des Präventionsparadoxes:

"A preventive measure that brings large benefits to the community offers little to each participating individual".

Das Präventionsparadox stellt ein grundlegendes Dilemma der bevölkerungs- und der risikogruppenbezogenen Prävention dar: Eine präventive Maßnahme, die für die Bevölkerung einen hohen Nutzen bringt, bringt dem einzelnen Menschen oft nur wenig. Umgekehrt hilft der Hochrisikoansatz Einzelnen, tut aber wenig, um die Krankheitslast (Burden of Disease, s. Kap. 10.1.2 und Kap. 10.1.5) in der Bevölkerung zu reduzieren. Dieses Dilemma ergibt sich immer dann, wenn das Risiko die Gesamtbevölkerung oder große Teile der Bevölkerung betrifft. Ein Beispiel hierfür wären Impfprogramme im Kindesalter, die zu Herdenimmunität (s. Kap. 9.1.3) führen, oder die Gurttragepflicht.

Aus Public-Health-Sicht ist eine bevölkerungsweite Intervention vielversprechender, weil sie mehr Krankheitsereignisse oder vorzeitige Todesfälle verhindern kann und die zugrundeliegenden Ursachen angeht. Solche Maßnahmen gehören oft zur Verhältnisprävention (s. Kap. 4.1.2) und sind politisch nicht immer leicht durch-

4 kardiovaskulär: das Herz und das Blutgefäßsystem betreffend 
zusetzten, da sie nicht selten mit kulturellen Normen und Gepflogenheiten oder handfesten wirtschaftlichen Interessen in Konflikt geraten. Die Hochrisikostrategie ist dagegen eine auf die Bedürfnisse der identifizierten Betroffenen zugeschnittene Verhaltensprävention (s. Kap. 4.1.2) und leichter umzusetzen. Die Betreuung erfolgt hier im Rahmen eines etablierten Verhältnisses zwischen dem Individuum und seinem Arzt oder seiner Ärztin (s. a. Kap. 4.4). Tab. 1.1 fasst die wichtigsten Merkmale sowie die Vor- und Nachteile der Hochrisiko- und der Bevölkerungsstrategie zusammen.

Die Hochrisikostrategie und die Bevölkerungsstrategie schließen sich selbstverständlich nicht gegenseitig aus, sondern können, wie von der WHO empfohlen, sinnvoll kombiniert werden. In vielen Situationen ist die Annahme eines Kontinuums nützlich, in dem Zwischenstufen zwischen den beiden Extremen bestehen. Schließlich können auch spezifische, auf eine Risikogruppe zugeschnittene Angebote sinnvoll sein, wie z. B. Anlaufstellen für drogenabhängige Personen, die diese mit sauberem Spritzenmaterial versorgen.

Tab. 1.1: Merkmale der Bevölkerungs- und der Hochrisikostrategie.

\begin{tabular}{|c|c|c|}
\hline & Bevölkerungsstrategie & Hochrisikostrategie \\
\hline Zielgruppe & Gesamte Bevölkerung & Risikogruppen \\
\hline Ansatz & Periphere (gesellschaftliche) Ursachen & $\begin{array}{l}\text { Unmittelbare (biologisch-individuelle) } \\
\text { Ursachen }\end{array}$ \\
\hline Vorteile & $\begin{array}{l}\text { Potentiell großer Nutzen für } \\
\text { die Bevölkerung } \\
\text { Beeinflusst zugrunde liegende Ursachen }\end{array}$ & $\begin{array}{l}\text { Intervention wird Individuum gerecht } \\
\text { Hohe Motivation von Betroffenen und } \\
\text { Gesundheitssystem } \\
\text { Politisch einfach umzusetzen }\end{array}$ \\
\hline Nachteile & $\begin{array}{l}\text { Nutzen für das Individuum wenig } \\
\text { sichtbar } \\
\text { Konflikte mit kulturellen Normen } \\
\text { oder wirtschaftlichen Interessen } \\
\text { Politisch schwierig umzusetzen }\end{array}$ & $\begin{array}{l}\text { Nutzen für Bevölkerung klein } \\
\text { „Symptombekämpfung“ } \\
\text { „Labeling“a und Medikalisierung der } \\
\text { Betroffenen }\end{array}$ \\
\hline Beispiele & $\begin{array}{l}\text { Reduktion des Salzgehalts in } \\
\text { verarbeiteten Lebensmitteln; } \\
\text { Tragen von Sicherheitsgurten im Auto; } \\
\text { Einschränkung des Zugangs } \\
\text { zu Schusswaffen; } \\
\text { Kampagnen zur Förderung des Konsums } \\
\text { von Früchten und Gemüse }\end{array}$ & $\begin{array}{l}\text { Medikamente oder Diätberatung zur } \\
\text { Senkung von Cholesterin, Blutdruck oder } \\
\text { Körpergewicht in der Primärprävention; } \\
\text { Interventionen der Sekundär- und } \\
\text { Tertiärprävention, z. B. Rückenschule bei } \\
\text { Menschen mit chronischen } \\
\text { Rückenschmerzen }\end{array}$ \\
\hline
\end{tabular}

a Labeling: Den betroffenen Personen wird pauschal eine bestimmte Eigenschaft zugeschrieben. 


\subsection{Public-Health-Ethik Matthias Egger, Lotte Habermann-Horstmeier}

Die Ethik ist eine angewandte Disziplin der Philosophie, die es sich zur Aufgabe macht, in verschiedenen Lebensbereichen Kriterien und Normen für gutes und richtiges menschliches Handeln zu entwickeln. Als Medizinethik bezeichnet man den Teilbereich der Ethik, der sich mit dem Handeln der verschiedenen Akteure in der medizinischen Versorgung, Pflege und Forschung beschäftigt. Ethische Fragen stellen sich in der Medizin immer wieder bei der Abwägung von Risiken oder Kosten bestimmter Diagnostiken und Therapien im Verhältnis zu ihrem Nutzen, aber auch im Zusammenhang mit dem Schutz nicht-einwilligungsfähiger Personen (z. B. Demenzkranke, Menschen mit geistiger Behinderung, Kinder oder Bewusstlose) oder bei der Planung und Durchführung klinischer Studien (s. Kap. 2.1.6). Besonders kontrovers diskutierte medizinethische Themen sind Sterbehilfe und Beihilfe zum Suizid, die z. B. in Deutschland und der Schweiz sehr unterschiedlich gehandhabt werden.

Die Public-Health-Ethik ist ein relativ neues Anwendungsgebiet der Ethik, das sich mit ethischen Fragen im Bereich der öffentlichen Gesundheitspflege beschäftigt. Solche Fragestellungen können z. B. bei der Durchführung von Überwachungs- und Kontrollmaßnahmen im Rahmen von Infektionskrankheiten ebenso auftreten wie bei der Durchführung von Screening-Programmen oder bei dem Erlass von Verboten (etwa des Rauchens in öffentlichen Räumen). Während in der Medizinethik die ArztPatient-Beziehung im Mittelpunkt steht, ist es in der Public-Health-Ethik das Verhältnis zwischen den staatlichen und nicht-staatlichen Institutionen einerseits und den BürgerInnen andererseits.

Ethik leitet ihre Prinzipien aus theoretischen Ansätzen ab. Eine der grundlegenden ethischen Theorien ist der Utilitarismus. Handlungen werden hier im Hinblick auf ihrer Konsequenzen bewertet, und zwar unter dem Gesichtspunkt der Steigerung des allgemeinen Wohlergehens. Dieser Ansatz ist dem Denken im Bereich Public Health sehr nahe. Die Daten, auf die man sich bei der Abwägung von Nutzen, Schaden und Kosten bestimmter Maßnahmen beruft, stammen in der Regel aus epidemiologischen und ökonomischen Studien (s. Kap. 2.1.5 und 2.5.1). Ein problematischer Aspekt des Utilitarismus ist, dass hier nur das Wohlergehen der Mehrheit berücksichtigt wird. Dem Utilitarismus wird deshalb das Konzept der Menschenrechte gegenübergestellt. Viele der 30 Artikel der Allgemeinen Erklärung der Menschenrechte, die die UNGeneralversammlung im Jahr 1948 verabschiedete, sind für Public Health relevant. Hierzu gehören z. B. der Anspruch auf den Schutz vor Diskriminierung, das Recht auf Fürsorge und Gesundheit, das Recht auf Bildung und das Recht, an der Gestaltung der öffentlichen Angelegenheiten seines Landes mitzuwirken. Erwähnt sind aber auch Pflichten gegenüber der Gesellschaft (s. Internet-Ressourcen auf unserer Lehrbuch-Homepage). 
Von besonderer Bedeutung im Hinblick auf einen Berufskodex sind die folgenden sechs wichtigen Prinzipien der Medizin- und der Public-Health-Ethik, die sich in einigen Bereichen überschneiden (s. Tab. 1.2).

Tab. 1.2: Übersicht über die wichtigsten ethischen Prinzipien im Bereich der Medizinethik und der Public-Health-Ethik.

\begin{tabular}{|c|c|}
\hline Medizin & Public Health \\
\hline Autonomie (Respect for autonomy) & Gegenseitige Abhängigkeit (Interdependence) \\
\hline $\begin{array}{l}\text { Jede Person ist frei in ihren Entscheidungen. Bei } \\
\text { medizinischen Maßnahmen oder der Teilnahme } \\
\text { an einer Studie muss die informierte } \\
\text { Zustimmung (Informed Consent) } \\
\text { der betroffenen Personen vorliegen. }\end{array}$ & $\begin{array}{l}\text { Das Handeln einer Person betrifft nicht nur } \\
\text { sie selbst, sondern auch andere Personen. } \\
\text { Jede Person ist auch von den Aktionen anderer } \\
\text { betroffen. }\end{array}$ \\
\hline Fürsorge (Beneficence und Non-maleficence) & Mitwirkung (Participation) \\
\hline $\begin{array}{l}\text { Schädliche oder riskoreiche Eingriffe } \\
\text { und Maßnahmen sollen vermieden werden. } \\
\text { Durch die Maßnahme/Studie wird das Wohl } \\
\text { der PatientInnen oder StudienteilnehmerInnen } \\
\text { gefördert. }\end{array}$ & $\begin{array}{l}\text { Public-Health-Maßnahmen werden unter } \\
\text { Mitsprache und mit dem Einverständnis } \\
\text { der betroffenen Bevölkerung geplant und } \\
\text { durchgeführt. }\end{array}$ \\
\hline Gerechtigkeit (Justice) & $\begin{array}{l}\text { Wissenschaftliche Abstützung } \\
\text { (Scientific evidence) }\end{array}$ \\
\hline $\begin{array}{l}\text { Das Prinzip der Gerechtigkeit fordert eine faire } \\
\text { Verteilung von Gesundheitsleistungen, Risiken } \\
\text { und Nutzen in der klinischen Forschung. }\end{array}$ & $\begin{array}{l}\text { Entscheidungen über Public-Health-Maßnahmen } \\
\text { sollen aufgrund von wissenschaftlichen Daten } \\
\text { und nicht auf der Basis von Annahmen und } \\
\text { Meinungen erfolgen. }\end{array}$ \\
\hline
\end{tabular}

Das Prinzip der Autonomie: Bei allen Maßnahmen im Bereich der Medizin und in Public Health muss die Entscheidungsfreiheit der betroffenen Menschen respektiert werden. Auch Public-Health-Maßnahmen bedürfen daher der informierten Zustimmung (Informed Consent, s. a. Kap. 2.1.6), z. B. im Zusammenhang mit Impfungen oder Screening-Untersuchungen.

Das Prinzip der gegenseitigen Abhängigkeit: Das Prinzip der Autonomie wird durch das Prinzip der gegenseitigen Abhängigkeit ergänzt und relativiert. Es besagt, dass das Handeln eines Einzelnen in der Regel auch andere Menschen betrifft. Eine mit HIV infizierte Person muss daher andere durch den Gebrauch von Kondomen vor einer Ansteckung schützen. Durch die Impfung des Krankenhauspersonals gegen die Virusgrippe (Influenza) können HochrisikopatientInnen vor Ansteckung geschützt werden (s. a. Kap 9.4.1). Ein Rauchverbot in öffentlichen Räumen ist u. a. aufgrund der schädlichen Wirkung des Passivrauchens (s. a. Kap. 4.2.2 und Kap. 6.4.3) gerechtfertigt. 
Das Prinzip der Fürsorge: Das Prinzip der Fürsorge beinhaltet die Verpflichtung, den Menschen Gutes zu tun und Schaden zu vermeiden. In Kombination mit dem Menschenrecht auf Gesundheit und Fürsorge lässt sich hieraus eine Verpflichtung des Staates ableiten, sich im Bereich Public Health zu engagieren. Hierzu gehört, dass die Lebensbedingungen der Menschen so gestaltet werden sollen, dass die einzelnen BürgerInnen Verantwortung für ihre Gesundheit übernehmen können. Dies ist in vielen Ländern bislang nicht der Fall (s. Kap. 10.2).

Das Prinzip der Mitwirkung: Dem Prinzip der Fürsorge steht das Prinzip der Mitwirkung gegenüber: Public-Health-Maßnahmen sollen stets unter Einbezug der betroffenen Bevölkerung geplant und umgesetzt werden. Handlungen, die gegen den Willen oder ohne die informierte Zustimmung der Betroffenen zu deren Wohl durchgesetzt werden (Paternalismus), widersprechen diesem Prinzip.

Das Prinzip der Gerechtigkeit: Gerechtigkeit wird in der Medizinethik vorwiegend als distributive Gerechtigkeit verstanden. Dies bedeutet, dass die Leistungen des Gesundheitssystems allen offen stehen und die Kosten fair auf die Mitglieder der Gesellschaft verteilt werden sollen (s. a. Kap. 3). Im Public-Health-Kontext leitet sich daraus die Verpflichtung ab, sozio-ökonomisch bedingte Ungleichheiten im Gesundheitszustand der Bevölkerung (s. Kap. 1.3.2) zu verringern. Es muss dabei darauf geachtet werden, dass sich die bestehenden Ungleichheiten nicht durch Public-Health-Maßnahmen, die vor allem von sozial Bessergestellten in Anspruch genommen werden, weiter vergrößern. Zum Prinzip der Gerechtigkeit gehört auch der Schutz vor Diskriminierung und Stigmatisierung.

Das Prinzip der wissenschaftlichen Abstützung: Die Vor- und Nachteile von PublicHealth-Maßnahmen sollen schließlich nicht von gesellschaftlichen Moralvorstellungen, Meinungen und Annahmen geleitet, sondern auf der Basis guter wissenschaftlichen Daten diskutiert werden (Prinzip der wissenschaftlichen Abstützung). Dieses Prinzip gilt sinngemäß auch für die Medizinethik, wird aber im Public-Health-Kontext besonders betont, weil hier oft gesunde Menschen betroffen sind.

Als erste Formulierung eines ethischen Berufskodex gilt der Eid des Hippokrates (s. Internet-Ressourcen), benannt nach dem griechischen Arzt Hippokrates von Kós (um 460 bis 370 v. Chr.). Im Jahr 1947 wurde der Nürnberger Kodex als Reaktion auf die während der Zeit des Nationalsozialismus im Namen der medizinischen Forschung begangenen Verbrechen verfasst. Er enthält ethische Richtlinien für die Durchführung von Experimenten am Menschen. Der Weltärztebund verabschiedete 1964 die Deklaration von Helsinki zu ethischen Grundsätzen für die medizinische Forschung am Menschen. Sie wurde seither mehrmals revidiert und dient heute den Ethikkommissionen als Grundlage für die Beurteilung klinischer Studien (s. a. Kap. 2.1.6). Im Bereich der Medizin haben ethische Diskussionen also anders als in Public Health 
schon eine lange Tradition. Die in den USA entwickelten ausführlichen Richtlinien für ethisches Handeln in Public Health (Principles for the Ethical Practice of Public Health) wurden von der American Public Health Association erst 2002 angenommen (s. Internet-Ressourcen).

Im Bereich Public Health werden in den letzten Jahren zunehmend auch ethische Fragen im Zusammenhang mit dem Thema Nudging diskutiert. Als Nudging bezeichnet man eine Methode, mit deren Hilfe man das Verhalten von Menschen auf vorhersagbare Weise zu beeinflussen sucht, und zwar ohne Verbote oder Gebote. So wäre es z. B. eine Nudging-Methode, Menschen zu veranlassen, in öffentlichen Gebäuden häufiger die Treppe anstatt der Rolltreppen zu nutzen, indem man die Treppe für das Publikum interessanter macht. Dieses Prinzip wurde u. a. im Bereich der Stockholmer U-Bahn angewandt. Hier wurden die Treppenstufen mit Klaviertönen unterlegt, sodass bei jeder Berührung ein Ton entstand. Die Passanten nutzten daraufhin die Treppenstufen wesentlich häufiger als die Rolltreppe. Von Kritikern dieser Methode wir jedoch immer wieder angeführt, dass es sich hierbei um eine Form des Paternalismus handelt. Zwar können die Adressaten einer solchen Maßnahme wählen, ob sie sie in Anspruch nehmen. Allerdings ist die Option bewusst so attraktiv gestaltet, dass es den Menschen schwerfällt, sie auszuschlagen. Ihnen ist damit in der Regel nicht bewusst, dass sie von einer bestimmten Gruppe (z. B. Public-Health-Fachleuten, Politikern oder auch der Werbebranche) in einer paternalistischen Weise dazu gedrängt werden, etwas zu tun, was sie sonst vielleicht nicht getan hätten. Im Hinblick auf die Prinzipien der Public-Health-Ethik lässt sich hier also durchaus darüber streiten, ob beim Nudging z. B. die Prinzipien der Autonomie und der Mitwirkung eingehalten werden.

\subsection{Public Health Genomics Albrecht Jahn, Nicole Probst-Hensch}

Public Health Genomics hat zum Ziel, genombasiertes Wissen und die dazu entwickelten Technologien mit Public-Health-Forschung, Gesundheitspolitik und Gesundheitsprogrammen zu verknüpfen (s. Office of Public Health Genomics).

Die Erkenntnis, dass genetische Faktoren bei der Entstehung und Manifestation vieler Krankheiten eine wichtige Rolle spielen, ist nicht neu. Von praktischer Bedeutung ist sie bisher vor allem bei monogenetischen Erkrankungen, wie z. B. der Phenylketonurie (PKU), bei der ein Enzymdefekt, verursacht durch eine Mutation in einem einzelnen Gen, dazu führt, dass sich die Aminosäure Phenylalanin im Körper der Betroffenen anreichert. Hierdurch kommt es zu einer schweren geistigen Entwicklungsstörung. Die frühzeitige Erkennung einer PKU über einen Bluttest bei Neugeborenen bietet die Möglichkeit, die Manifestation der Erkrankung durch eine phenylalaninarme Diät zu verhindern. Der Bluttest wird u. a. in Deutschland und der Schweiz 
im Rahmen des Neugeborenen-Screenings flächendeckend durchgeführt und gilt als ein Paradebeispiel für eine sinnvolle Screening-Maßnahme (s. Kap. 4.5.4). Seit einiger Zeit kann die PKU auch bereits vorgeburtlich mit Hilfe eines Gentests diagnostiziert werden. An solche genetischen Tests werden im Hinblick auf Sensitivität und Spezifität (s. Kap. 2.3.7) die gleichen Anforderungen wie an andere Tests im Bereich der klinischen Diagnostik und des Screenings gestellt.

Anders als die Genetik, die - wie im Beispiel der PKU - die Funktion einzelner Gene oder Gen-Kombinationen untersucht, befassen sich die Genomics mit dem gesamten Genom eines Organismus, um die Funktionen und Interaktionen von Genen und deren Produkten besser zu verstehen. Mit der Entschlüsselung des menschlichen Genoms im Jahr 2001 waren zunächst hochgesteckte Erwartungen im Hinblick auf medizinische Anwendungen der neuen Erkenntnisse verbunden. Diese Hoffnung hat sich insbesondere in Bezug auf die primäre und sekundäre Prävention nicht übertragbarer und chronischer Krankheiten bislang noch nicht in dem erwarteten Ausmaß erfüllt.

Derzeit versucht man, individuelle, auf genetischen Parametern beruhende, maßgeschneiderte Behandlungs- und Präventionsmaßnahmen zu entwickeln. Vertreter einer solchen ,personalisierten Medizin“ sprechen davon, dass sich in Zukunft auch das Fach Public Health weg von den klassischen, populationsbezogenen Konzepten, hin zu einer individualisierten Vorsorge und einer „Personalised Public Health“ entwickeln wird. Durch die Kenntnis ihrer genetischen Ausstattung könnten dann z. B. alle Menschen innerhalb einer Bevölkerung in die Lage versetzt werden, die für sie jeweils richtige Ernährungsweise zu wählen. Zur Analyse ihres Genotyps würden zuvor Testverfahren wie die Genomsequenzierung, DNA-Mikroarrays und die Schlüsseltechniken der Proteomik ${ }^{5}$ eingesetzt.

Diese Zukunftsvision berücksichtigt jedoch nicht, dass insbesondere im Bereich der chronischen, nichtübertragbaren Krankheiten (z. B. der Herz-Kreislauf-Erkrankungen [s. Kap. 8.2] und der bösartigen Tumore [s. Kap. 8.3]) nur wenige Krankheitsbilder durch ein einzelnes krankhaftes Gen verursacht werden. An der Entstehung der meisten Krankheiten sind mehrere Gene beteiligt. Darüber hinaus spielen hier auch komplexe Interaktionen zwischen Genen und Umwelt eine große Rolle. So können z. B. Faktoren, die prä- und perinatal auf den Menschen einwirken, an der Krankheitsentstehung (z. B. von Adipositas und Diabetes mellitus) mitwirken. Solche epigenetischen und non-genomischen ${ }^{6}$ Phänomene sind in der Lage, physiologische Abläufe im Körper eines Menschen bleibend $z u$ beeinflussen. Als epigenetische Veränderungen bezeichnet man Veränderungen von Zelleigenschaften, die nicht oder nicht permanent in der DNA festgelegt sind, jedoch trotzdem auf die Tochterzellen

5 Proteomik: Die Proteomik erforscht die in einer Zelle oder einem Individuum zu einem bestimmten Zeitpunkt vorhandenen Eiweiße (Proteine) mit biochemischen Methoden.

6 Non-genomisch: nicht auf genetischem Wege, nicht die Erbanlagen betreffend 
vererbt werden. Durch diese Veränderungen werden Chromosomenabschnitte oder ganze Chromosomen in ihrer Aktivität beeinflusst. Die Reihenfolge der NukleotidBausteine in der DNA ändert sich hierdurch jedoch nicht.

In der Vergangenheit hat sich die Untersuchung des Zusammenhangs zwischen genetischer Variabilität und Krankheitsrisiko vor allem auf Mutationen und Polymorphismen ${ }^{7}$ in spezifischen Genen beschränkt, von denen man annahm, sie hätten eine biologische Bedeutung (Kandidatengen-Ansatz). Da inzwischen die DNA von zahlreichen Menschen mit unterschiedlichem ethnischem Hintergrund genotypisiert und sequenziert werden konnte, kennt man heute eine Vielzahl von einzelnen Abweichungen in der Basenabfolge der DNA (= Single Nucleotide Polymorphisms $[S N P]$ ) und weiß, wie sie zueinander in Beziehung stehen. So können heute mit Hilfe von Genchips mehr als 1 Million Genvarianten, die über die ganze DNA einer einzelnen Person verteilt sind, schnell und kostengünstig bestimmt oder mit großer Wahrscheinlichkeit vorhergesagt werden. Im Rahmen von genomweiten Fall-Kontroll- oder Assoziationsstudien (Genome Wide Associations - GWA) wird so ohne vorher vorhandene Hypothesen nach typischen Mustern in Genen und Chromosomenregionen im menschlichen Erbgut gesucht, die mit der Entstehung einer spezifischen Krankheit in Beziehung stehen könnten. Bislang hat man schon eine Vielzahl von einzelnen SNPs gefunden, die auf ein erhöhtes Krankheitsrisiko hindeuteten. Die Resultate dieser Analysen geben wichtige und neuartige Hinweise auf relevante Krankheitsmechanismen. Von der Erforschung seltener Genvarianten und der genetischen Variabilität in molekularen Netzwerken ${ }^{8}$ sowie von der Untersuchung von Gen-UmweltInteraktionen ${ }^{9}$ erhofft man sich ein besseres Verständnis von Krankheitsrisiken, die möglicherweise modifizierbar sind und später vielleicht zu einer personalisierteren Risikovorhersage beitragen. Allerdings ist der Weg hin zu sinnvollen Gentests für die Vorhersage des Erkrankungsrisikos und für die Früherkennung einzelner Krankheiten noch weit. Zwar werden seit einiger Zeit v. a. über das Internet zahlreiche Tests erfolgreich vermarktet, die z. B. ein personalisiertes Präventions- und Diätkonzept versprechen. Brauchbare prädiktive ${ }^{10}$ diagnostische Tests, die auf dem Boden von Genom-Analysen entwickelt wurden, gibt es derzeit allerdings nur bei monogenetischen Erkrankungen. Da solche Erkrankungen jedoch lediglich einen geringen Anteil der gesamten Krankheitslast (Burden of Disease; s. Kap. 10.1.2) ausmachen, sind sie für Public Health nur von untergeordneter Bedeutung. Man erhofft sich aber von den deutlichen Fortschritten im Bereich der bioinformatischen und funktionellen Cha-

7 Polymorphismus: das Auftreten einer oder mehrerer Genvarianten innerhalb einer Population 8 Molekulare Netzwerke: Hierunter versteht man Wechselwirkungsnetzwerke auf molekularer Ebene, die das Zusammenspiel der Moleküle innerhalb einer Zelle umfassend beschreiben.

9 Gen-Umwelt-Interaktionen: Die individuelle genetische Ausstattung führt dazu, dass Umwelteinflüssen bestimmte Auswirkungen haben.

10 Prädiktiver genetischer Test: Gentest bei einer Person, die zum Zeitpunkt der Untersuchung noch keine Symptome einer Erkrankung zeigt 
rakterisierung (Annotation ${ }^{11}$ ) genetischer Varianten im Erbgut und von der Zusammenarbeit zwischen EpidemiologInnen, KlinikerInnen und GrundlagenwissenschafterInnen, dass auch die Resultate der genetischen Forschung im Bereich komplexer Erkrankungen in den nächsten Jahren zunehmend Eingang in den klinischen Alltag finden werden. Allerdings ist heute noch zweifelhaft, ob in Zukunft eine „stratifizierte Medizin“ möglich sein wird, bei der mit Hilfe von genetischen Tests gezielte Präventions- oder Therapieempfehlungen für Subpopulationen abgeleitet werden, die dann $\mathrm{zu}$ einer effektiveren und kostengünstigeren Prävention bzw. Therapie beitragen sollen.

Wie auf dem UN-Gipfel zu nichtübertragbaren Erkrankungen (2011) hervorgehoben wurde, kann ein wesentlicher Teil der vorzeitigen Krankheits- und Todesfälle aufgrund von Herz-Kreislauf-Erkrankungen, Erkrankungen der Luftwege, Krebs und Diabetes mellitus durch eine Reduktion weniger Risikofaktoren (Rauchen, übermäßiger Alkoholgenuss, fehlende Bewegung und ungesunde Ernährung) verhindert oder zumindest in ein höheres Alter verschoben werden. Die UN-Mitgliedsstaaten sind daher aufgefordert, entsprechende Maßnahmen durch gesetzliche Regulationen und Vereinbarungen mit der Nahrungsmittel- und Getränkeindustrie umzusetzen. Im Vergleich zu den hierdurch möglichen positiven Einflüssen auf die Krankheitslast weltweit leistet Public Health Genomics derzeit (noch) keinen wesentlichen Beitrag zu einem Gesundheitsgewinn auf der Bevölkerungsebene.

\section{Internet-Ressourcen}

Auf unserer Lehrbuch-Homepage (www.public-health-kompakt.de) finden Sie Links zu den hier verwendeten Quellen, $\mathrm{zu}$ weiterführender Literatur sowie $\mathrm{zu}$ anderen themenrelevanten Internet-Ressourcen.

11 Annotation: Funktionelle Zuordnung und Beschreibung z. B. der genauen Lage von Genen bzw. Genteilen; die Erkenntnisse können experimentell gewonnen sein oder aus Computer-gestützten Vorhersage stammen. 